\title{
Coda wave interferometry during the heating of deep geothermal reservoir rocks
}

\author{
Jérôme Azzola ${ }^{*} \mathbb{D}$, Luke Griffiths ${ }^{2}$, Jean Schmittbuhl ${ }^{1}$, Dimitri Zigone ${ }^{1}$, Vincent Magnenet ${ }^{3}$, Frédéric Masson ${ }^{1}$, \\ Michael Heap ${ }^{1}$ and Patrick Baud ${ }^{1}$
}

*Correspondence: jerome.azzola@unistra.fr ${ }^{1}$ Institut de Physique du Globe de Strasbourg, Université de Strasbourg/ EOST, Strasbourg, France Full list of author information is available at the end of the article

\begin{abstract}
Coda wave interferometry (CWI) is a high-resolution technique that aims at tracking small changes in a diffusive medium from the time correlation of seismic waveforms. CWI has been widely used in recent years to monitor the fine-scale evolution of fault zones and more recently of deep reservoirs. However, to provide a quantitative interpretation of the reservoir, direct modeling of physical effects like the influence of temperature on seismic wave scattering is required to investigate temperature effects from measurements of velocity changes. Here, we propose to quantify the impact of thermo-elastic deformation on CWI measurements by comparing experimental results obtained from a previous study on Westerly Granite to a numerical approach based on two combined codes (SPECFEM2D and Code_Aster) for modeling wave propagation in complex media during thermo-elastic deformation. We obtain two major results. First, we show that multiple reflections on the boundaries of our simplified numerical sample reproduce well the wave scattering properties of the experimental granitic sample characterized by a complex mineral assembly and a large set of microcracks. We based our comparison on the wave diffusion model that describes both the experimental and numerical samples (similarity in energy density function and mean free path). We also show that both samples share a similar thermo-elastic behavior, but only after the second heating and cooling cycle. Second, the stretching technique used for CWI measurements on both samples reveals reversible time shifts correlated with the thermo-elastic deformation of the sample. However, the influence of thermo-elastic deformation is different between our numerical proxy and the experimental sample. We discuss the role of irreversible deformation (e.g., microcracking) for the observed discrepancy by introducing temperature dependence of elastic moduli in the model. These results suggest that there are open perspectives to monitor thermal strain in geothermal reservoirs using CWI.
\end{abstract}

Keywords: Coda wave interferometry, Westerly Granite, Deep geothermal reservoir, Wave propagation simulation (SPECFEM2D), Thermo-elastic deformation simulation (Code ASTER) 


\section{Background}

At the laboratory scale, seismic methods, both active (ultrasonic wave velocity measurements) and passive (acoustic emission monitoring), provide a range of monitoring techniques to analyze the influence of temperature on a rock sample. In particular, coda wave interferometry (CWI) is a method that has recently been used to monitor thermal microcracking in granite (Griffiths et al. 2018). CWI uses scattered elastic waves observed in the late part of the seismogram (i.e., the coda) to track small changes in solid materials such as rocks (e.g., Poupinet et al. 1984; Ratdomopurbo and Poupinet 1995; Roberts et al. 1992; Snieder 2002). The late arrivals are indeed much more sensitive to small-scale perturbations as they intensively sample the medium through multiple scattering which leads to diffusive waves and equipartition (e.g., Ryzhik et al. 1996; Weaver 1982; Hennino et al. 2001; Snieder 2006). Crystals present in a granitic rock sample, for example, not only play a specific role in the thermal microcracking, but also act as many distinct scatterers, contributing to the diffusion of the wavefield propagating within the sample. This method is appropriate to follow both reversible phenomena such as elastic deformation, and irreversible phenomena such as thermo-elastic effects, which deeply impact the propagation of elastic waves and can thus be studied using late arrivals in the coda. For example, Grêt et al. (2006) used CWI on an Elberton Granite sample heated to $90{ }^{\circ} \mathrm{C}$ to measure the influence of temperature changes on ultrasonic wave velocity. They interpreted the arrival time perturbations measured in the coda of strongly scattered waves recorded while heating as an apparent decrease in wave velocity. This drop in apparent velocity presents a strong non-linear behavior when the sample was heated from 70 to $90{ }^{\circ} \mathrm{C}$, occurring contemporaneously with an increase in the acoustic emission rate, attributed to thermal microcracking. Consistent changes in rock microstructure have been recently monitored within a wider range of temperatures in Westerly Granite using the same CWI method (Griffiths et al. 2018). Westerly Granite is an interesting material for laboratory experiments as it has been extensively studied (e.g., Brace et al. 1966, 1968) and its physical properties are well known. The fine crystal size of Westerly Granite, about $1 \mathrm{~mm}$ in diameter, is similar to the so-called "two-mica granite" (e.g., Hooijkaas et al. 2006) which hosts the deep geothermal reservoir exploited at the Enhanced Geothermal System (EGS) test site in Soultz-sous-Forêts, France. Combining velocity measurements, acoustic emission monitoring, and high-resolution CWI measurements within a new experimental setup, Griffiths et al. (2018) monitored the microstructural evolution of Westerly Granite during multiple heating cycles from room temperature to $450{ }^{\circ} \mathrm{C}$. Interestingly, the study highlighted different mechanical behaviors between the first and the subsequent cycles. While large and mostly permanent changes in the waveforms were measured during the first cycle, interpreted as an apparent reduction in velocity with temperature, the reductions in wave velocity during the following cycles were lower in amplitude (Griffiths et al. 2018).

The influence of temperature changes on the physical properties of granite has been widely reported (Somerton 1992) and includes, for example, a decrease in elastic moduli (Heard and Page 1982; Griffiths et al. 2017), an increase in rock sample porosity (David et al. 1999), a reduction in strength (Nasseri et al. 2007; Chaki et al. 2008; Violay et al. 2017; Xu et al. 2017; Griffiths et al. 2017) and changes (generally an increase) in permeability (Géraud 1994; David et al. 1999; Darot and Reuschlé 2000; 
Chaki et al. 2008; Meredith et al. 2012). The influence of temperature variations on these mechanical parameters is more complex to understand when the rock sample is cyclically heated and cooled. This situation is, however, relevant for thermally dynamic environments such as geothermal reservoirs (e.g., sequences of mud circulations in wells during drilling, thermal stimulation of the wells, variations in well flow during exploitation, etc.). Indeed, the impact on the mechanical properties and characteristics of the rock may vary from one cycle to another. For instance, Thirumalai and Demou (1974) measured the thermal dilatation of two granites (Halecrest and Charcoal Granite) during cyclical heating and cooling. They observed a permanent dilatation of the granites following the first cycle, but the dilatation during the third cycle was reversible. During the first heating/cooling cycle, their observations show that microcracking occurred in the sample. The following cycles had a much-reduced impact on microcracking.

Understanding the influence of temperature changes on the physical and mechanical properties of reservoir rocks is an important issue for the monitoring of deep geothermal reservoirs such as Enhanced Geothermal Systems (EGS) (Olasolo et al. 2016; Lu 2018). One of the most representative rocks of the EGS reservoirs of the Upper Rhine Graben is granite. For example, at the Soultz-sous-Forêts site (France) (Gérard and Kappelmeyer 1987; Gérard et al. 2006), a fractured granitic reservoir (Genter and Traineau 1996; Dezayes et al. 2005; Hooijkaas et al. 2006; Ledésert et al. 2010; Villeneuve et al. 2018) is overlain by a $1.5 \mathrm{~km}$-thick sedimentary unit (Aichholzer et al. 2016; Griffiths et al. 2016; Heap et al. 2017; Kushnir et al. 2018a, b). The crystals and mineral inclusions in granite also play a specific role in its thermal response. Indeed, heating of a granitic rock results in the complex thermal expansion of its various minerals and leads to the build-up of thermal stresses that may induce thermal microcracking (Kranz 1983; Fredrich and Wong 1986; Griffiths et al. 2018). At a larger scale, thermal stimulation can promote the fracturing of a rock mass (Huenges and Ledru 2011). The increase in fracturing due to thermal stimulation will increase the permeability (e.g., Siratovich et al. 2015), and ultimately the efficiency, of the geothermal system. As the physical properties of rock are non-trivially influenced-at all scales-by changes in temperature and pressure, laboratory experiments on representative granite rock samples and the forward modeling of the involved processes are necessary to develop relevant seismic monitoring techniques and help to improve geothermal optimization.

The subject of the present study is the impact of thermal deformation on seismic wave diffusion and CWI measurements. For this purpose, we compare two distinctive approaches: the experimental results of Griffiths et al. (2018), and a numerical modeling of seismic wave diffusion during the same heating procedure. Indeed, the forward modeling of experimentally observed effects can provide a significant improvement in the understanding of physical phenomena. Numerical simulations of mechanical deformation allow the fine tuning of key parameters, such as mechanical properties or multi-step loading paths, which helps distinguish the various contributions to the complex mechanical response of the rock. Modeling is thus complementary to laboratory experiments, where measurements encompass the contribution of all processes at once and provides a tool to discuss the physical processes at the origin of the measurements. Such a forward modeling approach was recently used to highlight the effects of strain changes on CWI measurements (Azzola et al. 2018), raising questions on the relative 
contributions of various physical processes (velocity changes, heterogeneity of the elastic deformation field, amplitude variations, etc.) on CWI measurements. The modeling approach of Azzola et al. (2018) combined a spectral element approach (SPECFEM2D) to study the seismic wave propagation within a diffusive medium, while elastic deformation of the medium was modeled using a finite element code (Code_Aster) (Azzola et al. 2018).

We analyze the wave propagation properties of a medium that reproduce both acoustically and mechanically the measurements on the Westerly Granite rock sample during heating from room temperature to $450{ }^{\circ} \mathrm{C}$, with a specific focus on its thermo-elastic deformation. CWI of synthetic waveforms generated while heating or cooling are compared to laboratory measurements (Griffiths et al. 2018). The first section describes the principle of the methods developed in both studies. As a framework for the numerical modeling, the experimental method of Griffiths et al. (2018) is detailed first, as well as the experimental evolution of the waveforms during heating and cooling. The numerical scheme that models the experiment is presented as well as the principle of CWI in this methodology section. In "Results", results of the comparison of the experimental and numerical approaches are given for the wave scattering properties and the thermomechanical behavior of the samples. We then show the CWI signals measured at the laboratory and in the simulation, as a function of the sample temperature. The differences in behavior are discussed in "Results" on the basis of the dependence of mechanical parameters with temperature. Finally, conclusions are proposed in "Conclusions".

\section{Methods}

Summary of the experimental approach used by Griffiths et al. (2018) Laboratory sample description

Figure 1a presents the experimental apparatus used to measure changes in ultrasonic wave velocity during repeated heating and cooling cycles of a cylindrical sample $(20 \mathrm{~mm}$ diameter and $40 \mathrm{~mm}$ length) of Westerly Granite (from Rhode Island, USA). Westerly Granite was chosen as it is well-studied, and its physical and mechanical properties are well known and are near-isotropic (Lockner 1998). Furthermore, the physical, mechanical, and transport properties of Westerly Granite have been shown to exhibit permanent changes related to thermal microcracking when heated to temperatures of above $70{ }^{\circ} \mathrm{C}$ (Nasseri et al. 2007, 2009; Wang et al. 1989; Yong and Wang 1980; Griffiths et al. 2018).

\section{Laboratory setup description}

The rock sample was held between two vertical pistons made of heat resistant stainless steel (grade 310) and loaded using a LoadTrac II servo-controlled uniaxial press (Fig. 1a). A tube furnace with a constant temperature zone of $80 \mathrm{~mm}$ surrounded the sample. Further details of the experimental setup are provided in Griffiths et al. (2018).

The furnace was programmed to heat samples from room temperature to a maximum temperature of $450{ }^{\circ} \mathrm{C}$ (to remain within the operating range of the high temperature acoustic transducers), at a rate of $1{ }^{\circ} \mathrm{C} / \mathrm{min}$ and with a 2-h dwell time at $450{ }^{\circ} \mathrm{C}$, before cooling at the same rate. The sample was subject to three repeated heating and cooling cycles to investigate possible hysteresis effects. Wave velocity measurements were performed during each cycle. The sample temperature was measured using a thermocouple 
of diameter $1.5 \mathrm{~mm}$ inserted through a $1.7 \mathrm{~mm}$ hole drilled radially to the center of the sample.

During the thermal stressing of the sample, a pair of high temperature sensors used for acoustic measurements were in direct contact with opposing faces of the sample, as presented in Fig. 1a. The pair of acoustic sensors are considered as broadband, since they have a weak resonant frequency at $100 \mathrm{kHz}$ and an operating frequency range of $80-560 \mathrm{kHz}$. Using a signal generator, a $200 \mathrm{kHz}$ sinusoidal pulse was emitted every $50 \mathrm{~ms}$ at the upper transducer (source). The generator simultaneously triggered an acquisition card to record the pre-amplified voltage signal at the receiver (the lower transducer). $2 \mathrm{~ms}$ duration waveforms were recorded at a sampling rate of $2 \mathrm{MHz}$. Table 1 summarizes the parameters of the source receiver used in the setup. To ensure a constant coupling between the sensors and the sample during heating and cooling, both transducers were held in direct contact on the opposing faces of the granite sample using an axially applied force of $100 \mathrm{~N}$, equating to a uniaxial stress of approximately $0.3 \mathrm{MPa}$ on a $20 \mathrm{~mm}$ diameter sample.

Under ambient temperature conditions, measurements of the P-wave velocity, $v_{\mathrm{P}}$, and $\mathrm{S}$-wave velocity, $v_{\mathrm{S}}$, were performed using a separate, calibrated apparatus prior to, and following all three heating/cooling cycles using first arrivals (contrary to late arrivals in the coda for CWI). These measurements provided a reference for the velocity measurements. Measurements were made using two pairs of piezo-transducers in contact with

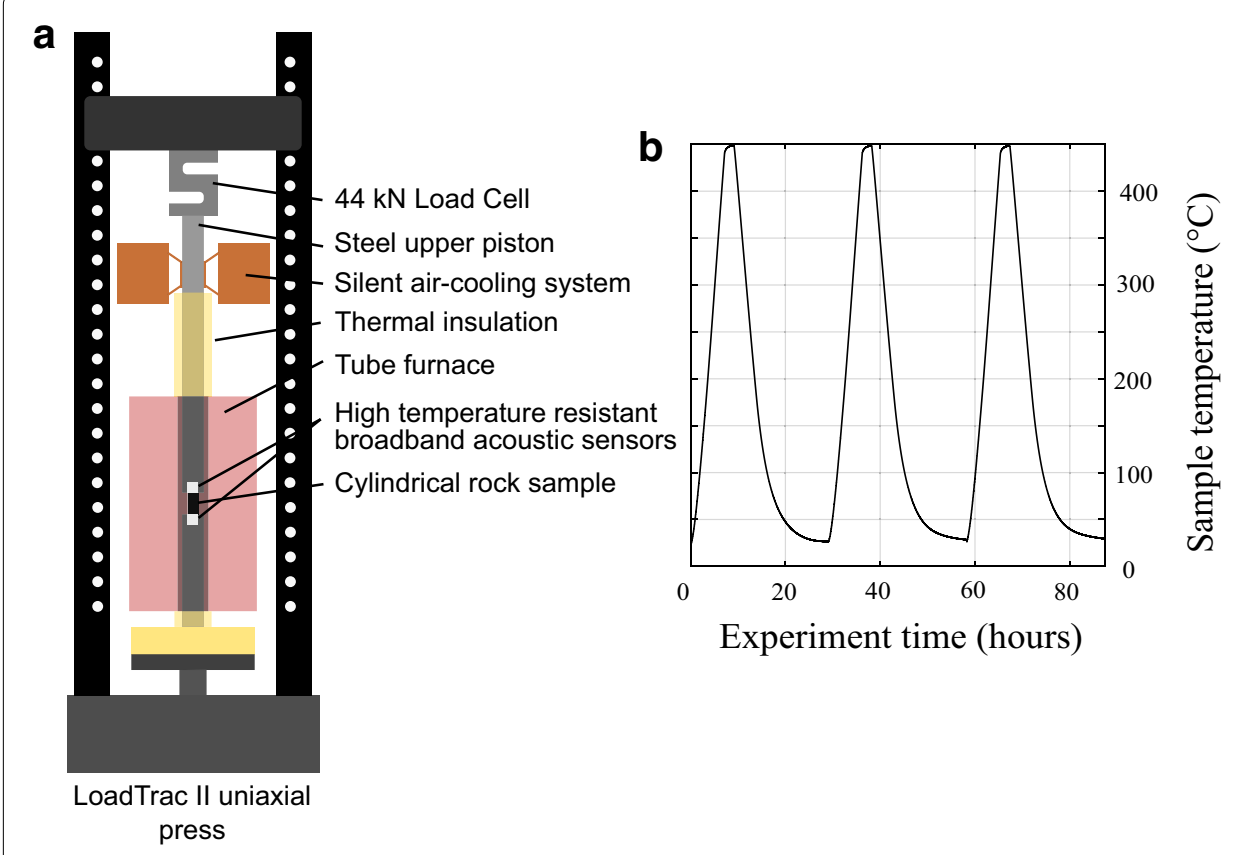

Fig. 1 a Sketch of the experimental setup (modified from Griffiths et al. 2018) for wave velocity measurements on a Westerly Granite sample. The apparatus consists of a uniaxial press, a tube furnace and for waveform measurements, a pair of high temperature resistant and broadband acoustic sensors that are set in direct contact with opposing ends of the sample [see Griffiths et al. (2018) for details]. Upper acoustic sensor is the source and the lower one is the receiver. $\mathbf{b}$ Evolution with time during three heating/cooling cycles, of the temperature measured at the center of the sample with a thermocouple inserted into a hole drilled radially 
Table 1 Parameters of the sensors and source function used in the laboratory experiments (S9215 high temperature sensors from Physical Acoustics) and in the simulation with heating and cooling rates

\begin{tabular}{|c|c|c|}
\hline & In laboratory & In simulation \\
\hline \multicolumn{3}{|l|}{ Source parameters } \\
\hline Temporal shape & Sinusoidal pulse & Ricker \\
\hline Central frequency $f_{0}$ & $200 \mathrm{kHz}$ & 200 kHz \\
\hline \multicolumn{3}{|l|}{ Receiver parameters } \\
\hline Record every & 17.5 s on average & Every loading step \\
\hline Length of signals & $2 \mathrm{~ms}$ & $2 \mathrm{~ms}$ \\
\hline Sampling rate & $2 \mathrm{MHz}$ & $1 \mathrm{GHz}$ \\
\hline $\mathrm{d} t$ & $5.0 \times 10^{-7}$ & $1.0 \times 10^{-9}$ \\
\hline \multicolumn{3}{|l|}{ Thermal load } \\
\hline Rate of cooling/heating & $1^{\circ} \mathrm{C} / \mathrm{min}$ & $3{ }^{\circ} \mathrm{C} / \mathrm{min}$ \\
\hline
\end{tabular}

opposing faces of the samples. One pair was oriented parallel to the sample length to measure P-wave first arrival times. The other, oriented perpendicular to the sample length, provided S-wave first arrival times. The source function was a sinusoidal pulse: $700 \mathrm{kHz}$ for P-wave, and within the range $100-500 \mathrm{kHz}$ for S-wave velocity.

\section{Numerical modeling of the experiment}

\section{Physical properties of the sample}

Our numerical simulations described below in "Results" intend to reproduce the experimental setup of Griffiths et al. (2018). The goal of the numerical analysis is to gain insight into the influence of temperature variation on the seismic wave velocity of granite. Our numerical approach focuses on the contribution, in the thermo-elastic domain, of reversible deformation. We also consider additional simulations designed to study the influence of temperature, a factor that greatly influences density, Young's modulus and bulk modulus, on the CWI measurements.

One of the main issues of the numerical approach is to introduce a numerical sample that accurately reproduces the macroscopic mechanical behavior of the real granite sample, as well as its wave diffusion properties while remaining as simple and homogeneous as possible. The dimensions of the numerical sample were chosen to be of same as the experimental sample $(40 \times 20 \mathrm{~mm})$. From wave velocity measurements carried out on the intact sample of Westerly Granite, at ambient temperature, we estimate macroscopic Poisson's ratio $v$ and to define $\mathrm{P}$ and S-wave velocities, respectively, noted $v_{\mathrm{P}}$ and $v_{\mathrm{S}}$. Young's modulus $E$, density $\rho$ and the thermal properties of the rock (isotropic thermal conductivity $\lambda$-thermal dilatation coefficient $\alpha$-specific heat capacity $c$ ) are chosen from Heard and Page (1982), Maqsood et al. (2004), Park et al. (2004), Dwivedi et al. (2008) and Schön (2011) to depict Westerly Granite. Measurements and mechanical parameters are presented in Table 2.

The wavelength used in the experimental work means that most of the scattering comes from multiple reflections on the boundaries of the sample, rather than reflections from crystal boundaries (Griffiths et al. 2018). This wave scattering behavior is reproduced in the numerical sample as we do not include details of the crystal assembly, but only use the multiple reflections at the free boundaries of the sample. 
Table 2 Elastic and thermal parameters of the medium of the simulation used in Code Aster and SPECFEM2D to reproduce the mechanical and acoustic behavior of the cored sample used in the experiments

\begin{tabular}{lll}
\hline $\begin{array}{l}\text { Wave speed (measured at ambient } \\
\text { temperature) }\end{array}$ & $v_{\mathrm{p}}\left(\mathrm{km} \mathrm{s}^{-1}\right)$ & 4.89 \\
& $v_{S}\left(\mathrm{~km} \mathrm{~s}^{-1}\right)$ & 2.70 \\
Thermal properties $\left(\right.$ at $\left.20^{\circ} \mathrm{C}\right)$ & Isotropic thermal conductivity $\lambda(\mathrm{W} / \mathrm{m} \mathrm{K})$ & 2.2 \\
& Isotropic thermal expansion coefficient $a\left(\mathrm{~K}^{-1}\right)$ & $12 \times 10^{-6}$ \\
& Specific heat capacity at constant pressure $\mathrm{C}(\mathrm{J} / \mathrm{kg} \mathrm{K})$ & 790 \\
Elastic properties & Young modulus $E(\mathrm{GPa})$ & 52 \\
& Poisson's ratio $\mathrm{V}$ & 0.28 \\
& Density $\rho\left(\mathrm{kg} / \mathrm{m}^{3}\right)$ & 2800
\end{tabular}

Young's modulus, density and the thermal properties of the rock are chosen from Heard and Page (1982), Maqsood et al. (2004), Park et al. (2004), Dwivedi et al. (2008) and Schön (2011)

The waveform recorded at a given receiver in the simulation is subsequently the contribution of many reflected waves that have travelled along multiple paths through the sample. The numerical sample is discretized using a $2 \mathrm{D}$ finite element mesh grid of 17244 elements with a characteristic length $\mathrm{lc}=0.5 \mathrm{~mm}$. This parameter corresponds to the characteristic distance between two neighboring nodes. The meshing of the sample is done using GMSH which is a GNU General Public License finite element mesh generator with 2-D quadrangle meshes using a Delaunay algorithm.

\section{Numerical scheme description}

The numerical simulation relies on combining two codes. The iterative thermo-mechanical deformation of the mesh grid is first obtained using the finite element software Code_Aster (EDF R\&D, general code for the study of the mechanical behavior of the structures). Each step of the loading process consists of the resolution of a linear thermal problem, which is combined to a mechanical loading that takes into account the $0.3 \mathrm{MPa}$ axial stress applied to the cored sample (Griffiths et al. 2018). We first solve the thermal problem according to the following equation:

$$
(\rho \cdot c) \cdot \mathrm{d} T / \mathrm{d} t+\operatorname{div}(-\lambda \cdot \operatorname{grad}(T))=0 .
$$

Temperature conduction within the sample is treated as an evolutionary regime. Figure 2 shows the evolution of temperature with simulation time computed by Code_Aster at the center of the sample. The insert on the right of Fig. 2 details the evolution during one step of the thermal deformation of the sample, when temperature is increased by $10{ }^{\circ} \mathrm{C}$ from 200 to $210{ }^{\circ} \mathrm{C}$. For each thermal step, the initial state is a homogeneous temperature field $T=T_{\mathrm{i}}$, and the thermal loading is an imposed temperature on the edges of the sample $T=T_{\mathrm{i}}+10^{\circ} \mathrm{C}$. The insert of Fig. 2 shows how temperature diffuses with time during a given loading step and shows that the stabilization time is about $100 \mathrm{~s}$. It also shows that the temperature field is homogenous to $10^{-3}{ }^{\circ} \mathrm{C}$ following $200 \mathrm{~s}$, which justifies the initialization of each thermal step by a homogeneous field. This heating rate (of about $3{ }^{\circ} \mathrm{C} / \mathrm{min}$; Table 1 ) is necessary for the stabilization of temperature at the end of each step of the simulation, in line with the quasi-thermal equilibrium in the laboratory experiments (heating rate of $1{ }^{\circ} \mathrm{C} / \mathrm{min}$; Table 1 ). 


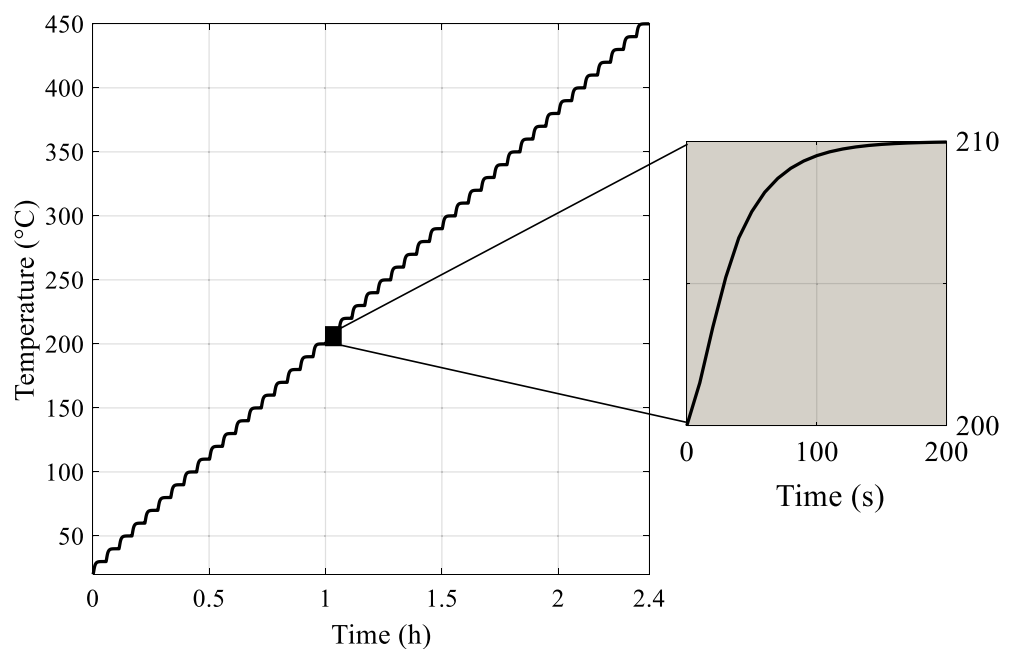

Fig. 2 Evolution of the sample temperature with time in the simulation. The temperature is computed at the middle of the sample using Code_Aster, while temperature is increased step by step from 20 to $450^{\circ} \mathrm{C}$ by $10^{\circ} \mathrm{C}$ steps. Right inset shows the progressive diffusion of temperature during one step of the deformation process in the simulation (from 200 to $210^{\circ} \mathrm{C}$ )

Equation (1) includes a dependency of the density with the temperature. We first recall that density is directly related to the volumetric deformation $e_{\mathrm{vol}}$ :

$$
e_{\mathrm{vol}}=\delta \mathrm{Vol} / \mathrm{Vol}_{0}=-\delta \rho / \rho_{0} .
$$

Second, S-wave speed $v$ and density $\rho$ are linked by

$$
v=\sqrt{3 \cdot K \cdot E / \rho \cdot(9 \cdot K-E)},
$$

where $K$ is the bulk modulus and $E$ is the Young's modulus, are assumed constant in the simulation, in the thermo-elastic domain. As a consequence, a volumetric change of the medium during heating or cooling results in an increase in density, and subsequently in a velocity change. To discuss this influence of the medium density changes on the CWI measurements, the variation of relative time delays with volumetric strain are analyzed in "Results".

The numerical scheme is based on the chaining of a thermal and a mechanical problem. As a result, we solve the mechanical problem according to the following equation:

$$
\operatorname{div}\left[C\left(e_{\mathrm{tot}}-\alpha \cdot\left(T-T_{\mathrm{i}}\right)\right)\right]=0,
$$

with $C$ the elasticity tensor, $\alpha$ the thermal dilatation, $e_{\text {tot }}$ the total deformation, $T$ and $T_{\mathrm{i}}$ the temperature and reference temperature of $25^{\circ} \mathrm{C}$. We neglect the effects of gravity in Eq. (2). The calculations carried out with Code_Aster result in a displacement field at the nodes of each element. At each step, the current mesh grid is then deformed accordingly to the computed displacement field.

The updated mesh grid is then an input of SPECFEM2D (a 2D spectral element code; see Komatitsch and Vilotte 1998). It simulates the wave propagation in the medium from the emitted source assuming free boundaries. A Ricker Wavelet of dominant frequency $f_{0}=200 \mathrm{kHz}$ (see top-right inset of Fig. 3) is sent in the medium 

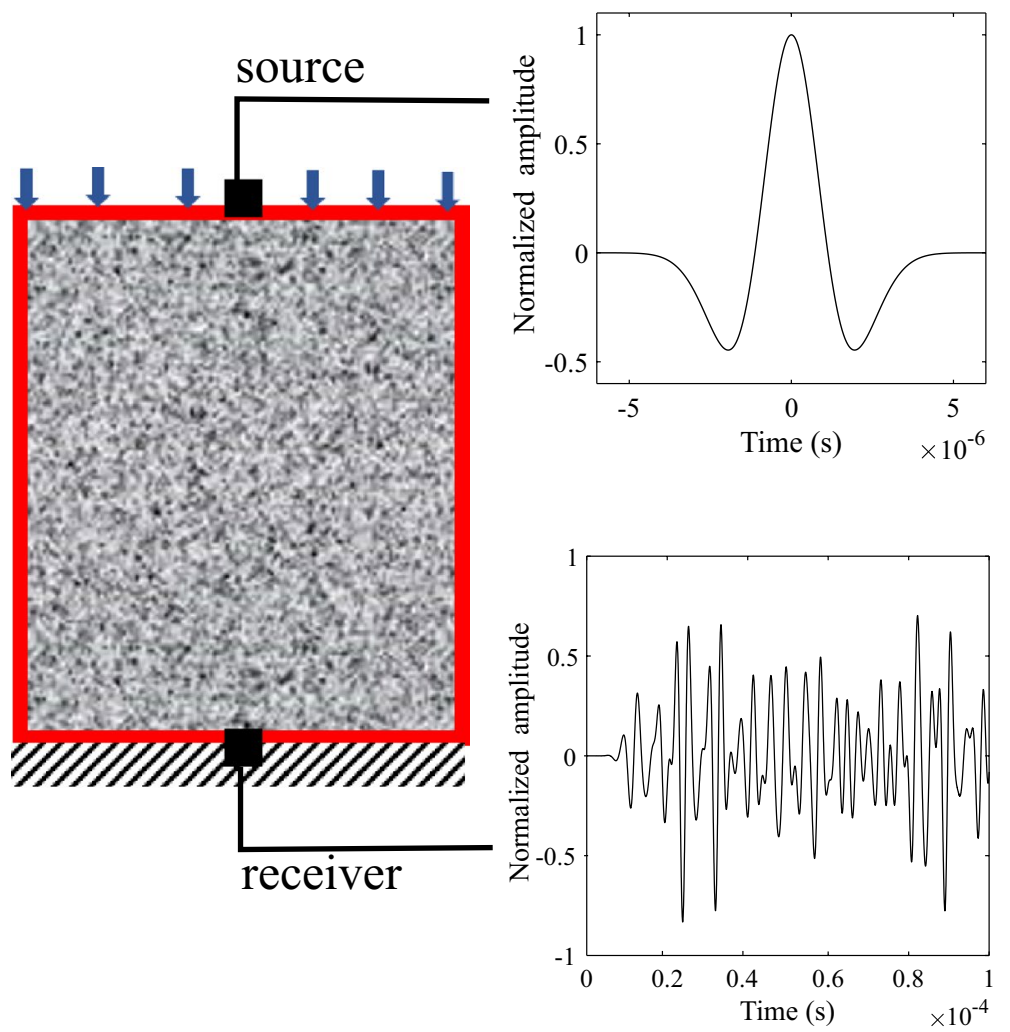

\section{- Mechanical loading $0.3 \mathrm{MPa}$ UII/, No displacement}

\section{- Thermal loading}

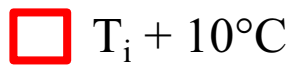

Fig. 3 Illustration of the principle of the numerical approach. Using a finite element method (Code_Aster), we apply a thermal and mechanical loading, step by step, to the numerical sample chosen to be of same size as the experimental sample $(40 \times 20 \mathrm{~mm})$. During each step, the mechanical loading is a $0.3 \mathrm{MPa}$ vertical stress and the thermal loading is a constant temperature at the borders. Temperature is increased by steps of $10{ }^{\circ} \mathrm{C}$. The wave propagation is computed using a spectral element method (SPECFEM2D). A Ricker wavelet (see top-right insert) is emitted in the medium from the source (top) and recorded at the opposite side (bottom) by a virtual 32-sensor receiver array

from the source and recorded at the opposite side by a 32-sensor long receiver line (see bottom-right insert of Fig. 3 for an example of recorded seismogram at a given receiver position). Absolute locations of the sources and receivers remain unchanged from the onset to the end of the deformation process. Table 1 details the parameters of the source-receiver pairs used in both the laboratory and in the simulation. The numerical approach that we developed allows us to test the effect of the elastic deformation of the sample independently of intrinsic wave velocity variations. When focusing in a first approach on the influence of reversible deformation in the thermoelastic domain, wave velocities in all elements are kept constant. The only parameter evolving during successive steps is the mesh grid. In laboratory experiments, the effects of reversible elastic deformation are in general difficult to distinguish from intrinsic variations that are related to anelastic and irreversible processes like damage 
development (Snieder 2006). In a second approach, bulk and Young's modulus, density and thereby elastic wave velocities depend on temperature, which enables us to discuss the partitioning between each effect. The flexibility of the numerical approach complements the laboratory experiments and compensate for its limits.

In the following sections, the "reference" seismogram refers to the one recorded at ambient temperature $T=T_{0}$, and the "perturbed" seismogram refers to the one recorded at a given temperature. CWI is used to compare these perturbed seismograms with the "reference" one to analyze changes induced by the thermo-elastic deformation.

\section{Coda wave interferometry (CWI)}

Coda wave interferometry aims to track small velocity changes in solid materials, such as rocks, where elastic waves are strongly diffused. As a result of the strong diffusion, coda waves intensively sample the medium through multiple scattering, such that the travel time delays measured between "reference" and "perturbed" waveform taken in the coda are much more sensitive to slight perturbations in the medium than in the early part of the waveform (e.g., Poupinet et al. 1984; Snieder 2006; Campillo and Roux 2015). Changes occurring in the medium-which include structural changes, the displacement of scatterers or mechanical deformation of the boundaries-delay the travel time of these scattered waves (Stehly et al. 2007), which make them observable using stretching or correlation methods.

\section{Description of the coda wave interferometry techniques}

The CWI measurements performed in the laboratory and in the simulation are based on a stretching approach of the whole signal, the first arrivals being a negligible part of the signal. Sens-Schönfelder and Wegler (2006) proposed to estimate the relative time differences $\varepsilon=\delta t / t$ in the late part of the signal between two successive acquired waveforms, as the factor by which the time axis of one of the traces must be stretched or compressed to obtain the best correlation with the other trace. In this technique, one defines "stretched" versions of one of the waveform of the pair such as $S(t)=R((1+a) t)$, with a given coefficient of stretching $a$. Following Sens-Schönfelder and Wegler (2006) and Larose and Hall (2009), we use a spline interpolation in the time domain to stretch the waveform. We then calculate the cross-correlation function between the other unstretched waveform of the pair with several stretched waveforms. The relative time shift-i.e., the relative variation of delay with time, assuming a linear variation of the delays in the coda-is equal to the value of a corresponding to the maximum of the cross-correlation function. This method is applied to the first $0.5 \mathrm{~ms}$ of the seismograms.

\section{Application to laboratory and numerical results}

The significant perturbation of the medium during the thermal loading induces a significant evolution of the waveforms and leads to a weak correlation between the first recorded waveform, the reference, and subsequent waveforms recorded during heating. Accordingly, it is not possible to apply the stretching technique using the first waveform as a unique reference. As a result, the waveform recorded at a given temperature $T=T_{k}$ is constantly compared with the one previously measured at $T=T_{k-1}$, which yields the incremental relative time shift noted for the following equation $\varepsilon\left(T_{k} T_{k-1}\right)$. The global 
relative time shift $\varepsilon\left(T_{i}, T_{0}\right)$ is obtained from the comparison of the waveform recorded at a given temperature $T_{i}$, with respect to the reference waveform, recorded at temperature $T_{0}$, according to Eq. (5), from a scaling factor and the preceding relative time shifts $\varepsilon\left(T_{k}\right.$, $\left.T_{k-1}\right)$ :

$$
\varepsilon\left(T_{k}, T_{0}\right)=\prod_{i=1}^{k}\left(\varepsilon\left(T_{i}, T_{i-1}\right)+1\right)-1 .
$$

In the numerical simulation, waveforms are compared in pairs using a stretching approach along the $2 \mathrm{~ms}$ long signal (Sens-Schönfelder and Wegler 2006). Since the simulated waveforms are weakly perturbed by the thermal loading, they are all compared to the initial waveform (not incrementally as in the experiments). We verified that the incremental comparison of pairs of simulated waveforms and their comparison to a common reference led to identical CWI results.

The receiver line used in the numerical modeling is a 32-sensor long array. Seismograms are thus acquired on distinctive receivers equally distributed along the bottom of the sample for each wave propagation modeling. This specificity of the numerical model enables us to analyze the spatial variability of our numerical measurements, given that CWI methods are applied at each receiver. When comparing a given pair of waveforms by stretching, we calculate the dispersion of the 32 distinctive relative time shifts estimations as well as the mean relative time shift. The fluctuations in the measurements are minor: the standard deviation of this population typically represents $8 \%$ of the mean value. It demonstrates the spatial homogenization occurring in our sample due to the diffraction of the wavefield. As a result of this spatial homogenization, the relative time shift measured in our numerical approach at each step of the thermal loading of the sample is the average of the 32 distinctive estimations.

\section{Results}

\section{Comparison of the wave scattering properties}

We check here that the numerical sample reproduces the acoustic behavior of the granite sample used in the laboratory experiments. For this, we compare the scattering properties of the numerical sample and the granite sample, by computing the energy density $W(r, t)$ for both the experimental and numerical records in the same manner as Wegler and Lühr (2001) according to the following equation:

$$
W(r, t)=\sum_{i=1}^{3}\left(f_{i}^{2}+H\left(f_{i}\right)^{2}\right) .
$$

Here, $f_{i}$ (in $\mathrm{m} \mathrm{s}^{-1}$ ), for $i$ varying from 1 to 3 , are the components of the seismogram measured in the simulation and in the laboratory experiments at ambient temperature. $H$ is the Hilbert transform. The observed energy density function is then compared to the wave diffusion model for body waves. This allows us to estimate the mean free path $l$ by least square fitting, as proposed in Olivier et al. (2015). The mean free path is the mean distance between two consecutive diffusion events and is a key parameter to assess the development of the diffusive wavefield in both propagation 
media. As shown in Nakamura (1977) and Dainty and Toksöz $(1977,1981)$, the diffusion model predicts the seismic energy of multi-scattered body waves $W(r, t)$ as a function of time and space:

$$
W(r, t)=E_{\mathrm{S}} \cdot\left(\frac{4 \cdot \pi \cdot v_{\mathrm{s}} \cdot t}{3 \cdot \eta_{\mathrm{s}}}\right)^{-\frac{3}{2}} \cdot e^{\left(-\eta_{\mathrm{i}} \cdot v_{\mathrm{s}} \cdot t-\left(\frac{3 \cdot r^{2} \cdot \eta_{\mathrm{s}}}{4 \cdot v_{\mathrm{s}} \cdot t}\right)\right)},
$$

where $W(r, t)$ is the energy density, $E_{\mathrm{s}}$ the source energy, $v_{\mathrm{S}}$ the S-wave velocity, $r$ the distance to the source, $\eta_{\mathrm{i}}$ the intrinsic attenuation defined as the inverse of the intrinsic absorption characteristic length $l_{\mathrm{a}}$ and $\eta_{\mathrm{s}}$ the scatter attenuation which is the inverse of the mean free path $l$. Equation (7) is linearized by choosing an adequate reference in time $t_{1}$ and distance $r_{1}$ so that $W_{1}\left(r_{1}, t_{1}\right)=1 \mathrm{~J} \mathrm{~m}^{-3}$, and by taking the natural logarithm of a referenced function $U(t)$ defined in Eq. (8). Three dimensionless coefficients $a_{1}, a_{2}$ and $a_{3}$ appear in Eq. (8) and are expressed with parameters of the model (Wegler and Lühr 2001):

$$
\operatorname{Ln} U(t)=a_{1}+a_{2} \cdot t+\frac{a_{3}}{t} .
$$

$$
\text { with } \quad U(t)=\left(\frac{W(r, t)}{W_{1}}\right) \cdot\left(\frac{t}{t_{1}}\right)^{\frac{3}{2}}
$$

$$
\text { and } a_{1}=\ln \left(\frac{E_{0}}{W_{1}}\right) \cdot \frac{4 \cdot \pi \cdot v_{\mathrm{s}} \cdot t_{1}}{\left(3 \cdot \eta_{\mathrm{s}}\right)}{ }^{-\frac{3}{2}} ; \quad a_{2}=\eta_{\mathrm{i}} \cdot v_{\mathrm{s}} ; \quad a_{3}=-\frac{3 \cdot \eta_{\mathrm{s}} \cdot r^{2}}{4 \cdot v_{\mathrm{s}}} \text {. }
$$

It appears that $a_{3}$ is expressed as a function of the intrinsic attenuation. Intrinsic attenuation is the inverse of the mean free path, which enables us to estimate the mean free path from $a_{3}$ :

$$
l=\eta_{\mathrm{s}}^{-1} .
$$

The observed energy density function $W(r, t)$ is linearized by multiplying Eq. (3) with the geometric factor $t^{3 / 2}$ and by taking the natural logarithm of both sides, which allows us to reconstruct the left side of Eq. (8). Figure 4 shows the logarithmic representation of the energy density functions computed with the first $0.3 \mathrm{~ms}$ of the original waveforms recorded at the laboratory and in the simulation. The comparison of these functions shows that the numerical medium used in the simulation describes the wave scattering occurring in the experimental granite sample. The comparison presented in Fig. 4 shows that a longer time is required to obtain a diffuse wavefield in the medium than in the real granite sample. Grain inclusions that contribute to diffuse the wavefield in addition to the multiple reflections in the real granite sample could explain this difference in behavior.

Dimensionless coefficients $a_{1}, a_{2}$ and $a_{3}$ are estimated using a least square fitting from Eq. (8). With $v_{S}=2700 \mathrm{~m} \mathrm{~s}^{-1}$ and $r=40 \mathrm{~mm}$, we finally estimate the mean free path using Eq. (9). Figure 4 shows in addition the model obtained by a least square 


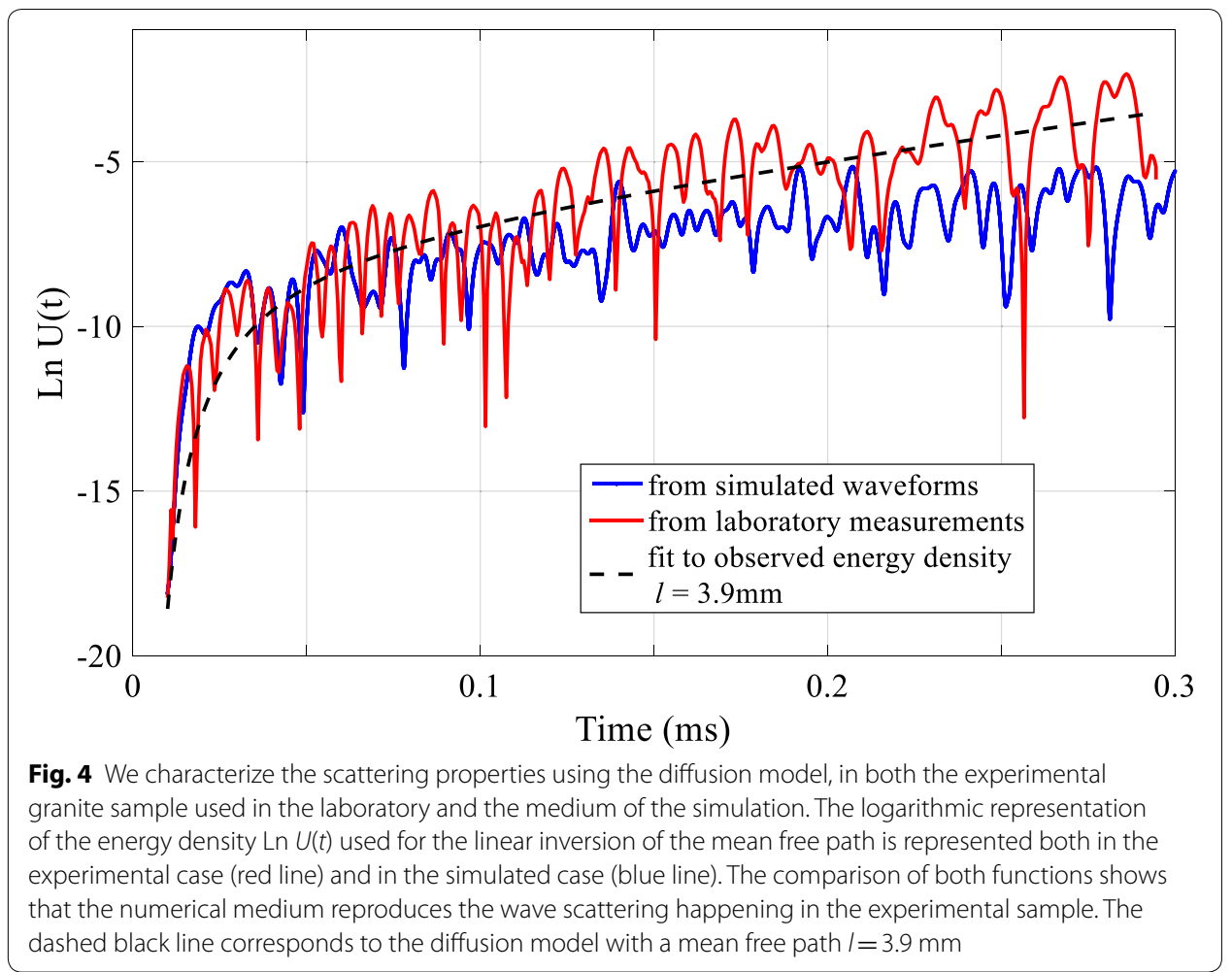

fitting of the linearized energy density functions Ln $U(t)$ computed from the laboratory results. The mean free path is estimated to be $l=3.9 \mathrm{~mm}$.

The application of CWI techniques requires the strong scattering regime to be established. In multi-scattering regimes, the wave front emitted from the source sees the heterogeneities several times before reaching the receiver. The condition necessary to assess strong scattering is that the mean free path $l$, the size of the defects $d$, the size of sample $D$ (here, $40 \mathrm{~mm}$ ), and the wavelength $\lambda$ are satisfying the inequality: $\lambda \leq d \leq l \leq D$ (Planès and Larose 2013). In our setup, we calculated a mean free path $l=3.9 \mathrm{~mm}$. The wavelength $\lambda=12 \mathrm{~mm}$ is estimated using the central frequency of the Ricker source and the S-wave velocity. This shows that the inequality and the requirements for strong scattering are not satisfied. Meanwhile, both linearized energy density functions exhibit strong similarities: the medium of the simulation properly describes the scattering that occurred in the experimental sample. To fulfill the condition of strong scattering, the laboratory experiments and the simulation would have required a source of higher frequency. To gain insight on the consequences of not verifying the inequality on the simulated and laboratory CWI results, we ran simulations with a Ricker source of higher frequencies: $f_{0}=800 \mathrm{kHz}$ and up to $f_{0}=1000 \mathrm{kHz}$. In each of these simulations, the calculated wavelength (respectively, $\lambda=3 \mathrm{~mm}$ and $\lambda=2.4 \mathrm{~mm}$ ) and the revalued mean free path satisfy the condition for strong scattering. With $f_{0}=800 \mathrm{kHz}$, the model obtained by least square fitting corresponds to an estimated mean free path $l=5.6 \mathrm{~mm}$ and with $f_{0}=1000 \mathrm{kHz}$, it is $l=5.3 \mathrm{~mm}$.

Figure 5 shows the simulated CWI measurements obtained for the three central frequencies as well as the linear regressions fitted to the measurements. It shows that the 


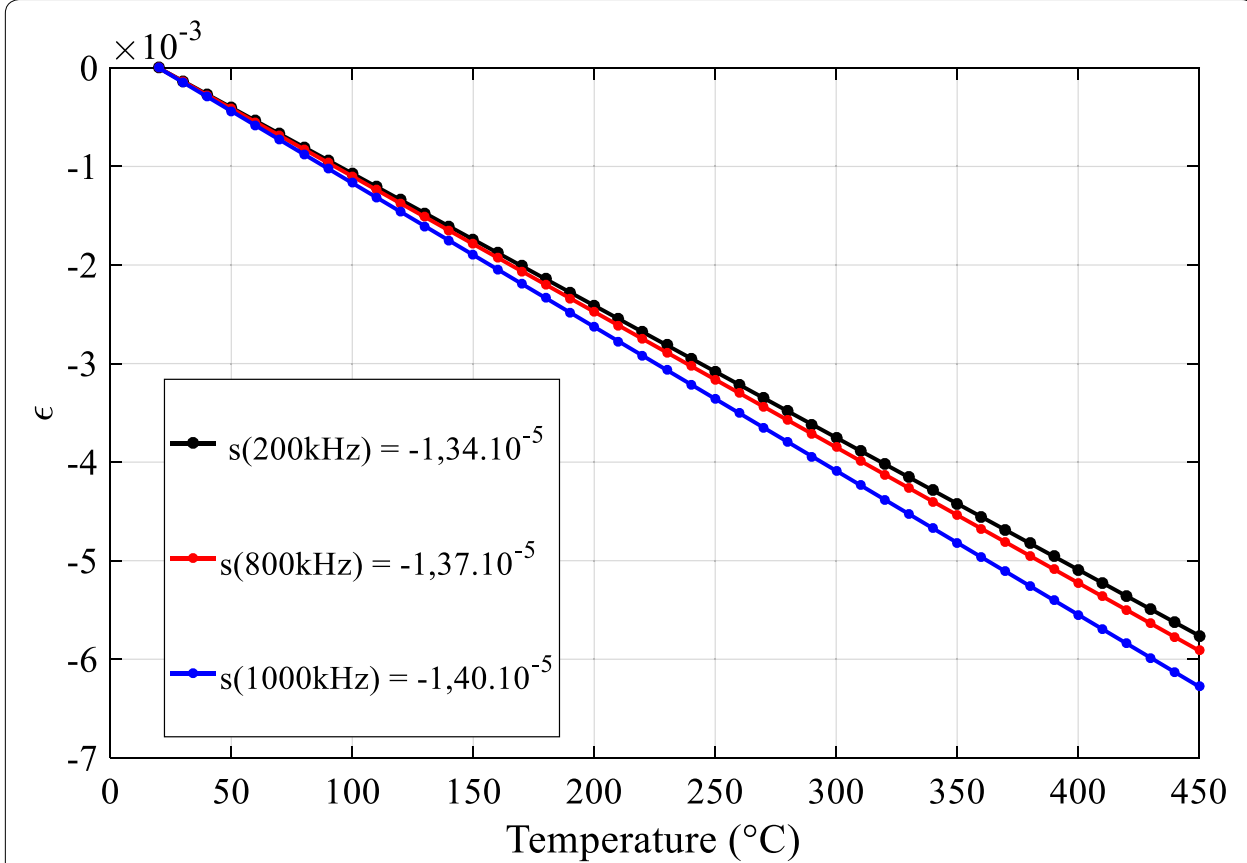

Fig. 5 Comparison of simulated CWI measurements obtained for three different central frequencies $\left(f_{0}=200 \mathrm{kHz}, f_{0}=800 \mathrm{kHz}\right.$ and $\left.f_{0}=1000 \mathrm{kHz}\right)$ of the Ricker-shaped source, for which the condition necessary to assume a strong scattering regime is verified. Relative time delays are measured by the "stretching technique" when comparing the waveform recorded at a given temperature with respect to the reference waveform. A linear regression is fitted to the measurements to estimate the slopes $s\left(f_{0}\right)$

slope of the linear regression at higher frequencies $s\left(f_{0}=800 \mathrm{kHz}\right)$ and $s\left(f_{0}=1000 \mathrm{kHz}\right)$ present less than $5 \%$ of variations compared with the reference $s\left(f_{0}=200 \mathrm{kHz}\right)$. This test demonstrates that, even if the inequality for strong scattering is not perfectly fulfilled for $f_{0}=200 \mathrm{kHz}$, it is possible to extract relevant information from the diffuse wavefield at that central frequency.

\section{Comparison of the mechanical behavior}

The numerical sample intends to reproduce the mechanical behavior of the granite sample used in the laboratory experiments. In the simulation, we focus on the elastic deformation of the sample when the temperature is progressively increased from 20 to $450{ }^{\circ} \mathrm{C}$ in $10{ }^{\circ} \mathrm{C}$ increments. The mesh grid, which is an input for the wave propagation simulation, is progressively deformed. As the mechanical parameters are considered constant with temperature, the numerical scheme intends to test the effect of the material deformation (in particular of thermal dilatation of the sample), independent of intrinsic perturbations related to inelastic deformation (microcracks, plastic deformation, etc.).

The volumetric deformation is computed as the trace of strain tensor (obtained from Code_Aster): volumetric strain $e_{\mathrm{vol}}$ is the sum of the components $e_{\mathrm{YY}}$ and $e_{\mathrm{XX}}$ of the strain tensor. Figure 6 shows three maps of the volumetric deformation, at the beginning of the heating process $(t=10 \mathrm{~s})$, while temperature diffuses $(t=50 \mathrm{~s})$ and after stabilization of the temperature ( $t=170 \mathrm{~s})$, in the simulation. Each map of the volumetric deformation (top panels in Fig. 6) is associated with the corresponding map of the temperature field 


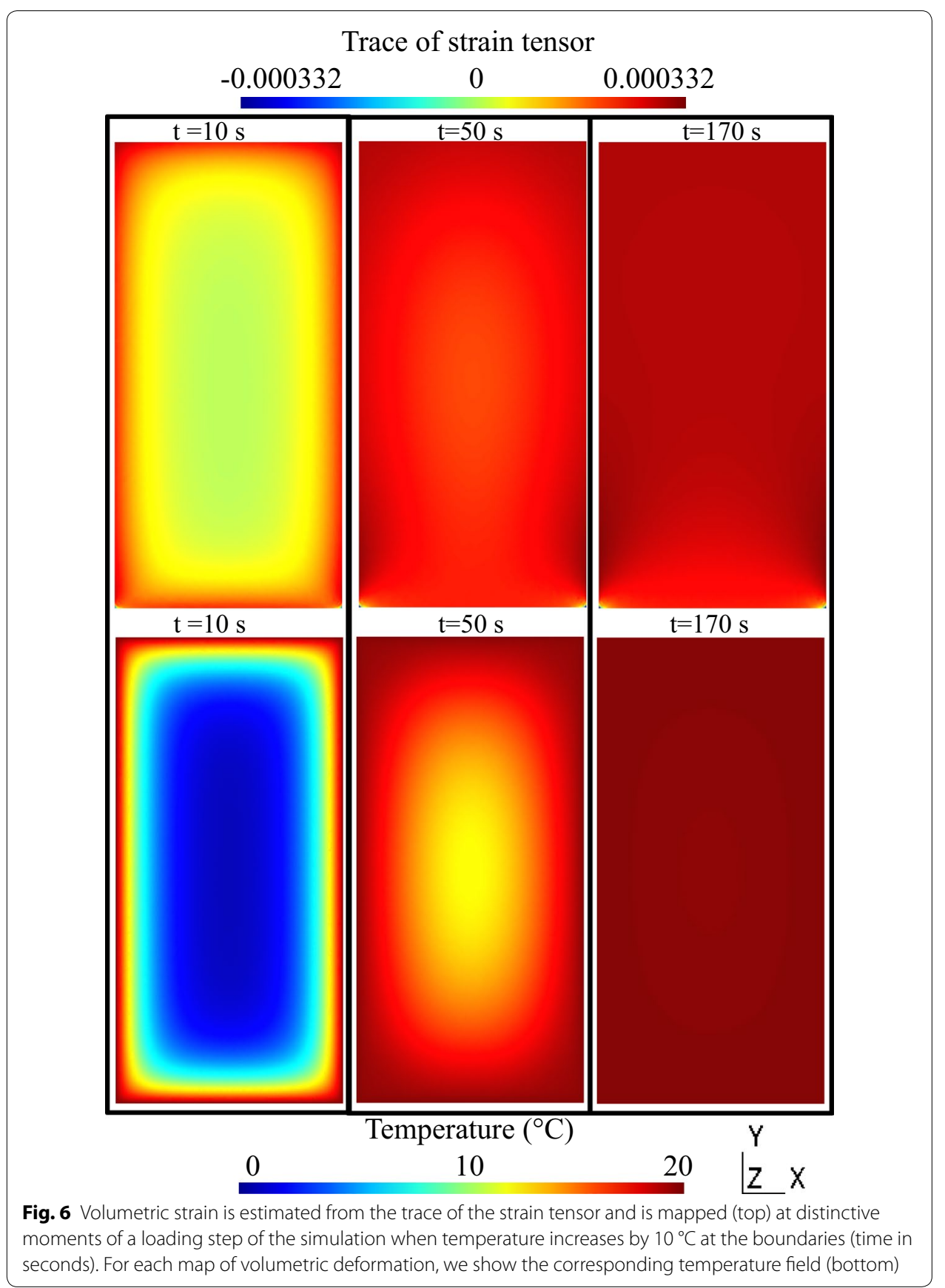

(bottom panels). The temperature, recorded at the center of the sample each time a $10{ }^{\circ} \mathrm{C}$ increment is set at its boundaries, is also represented in Fig. 3.

The temporal evolution of the temperature field shows the progressive diffusion of heat within the sample, from the moment the boundary conditions are applied at the boundaries of the sample to the homogenization of the temperature field. The temperature field and the volumetric deformation stabilize after about $100 \mathrm{~s}$. This duration is consistent with the characteristic time of diffusion $\tau=e^{2} / D$ computed from the characteristic length of diffusion e and the thermal diffusivity $D$. According to the parameters 
chosen in Table 2, $D=\lambda / \rho \cdot c=10.0 \times 10^{-7} \mathrm{~m}^{2} \mathrm{~s}^{-1}$. Considering the size of the sample $(40 \times 20 \mathrm{~mm})$ and the temperature increase imposed at its boundaries, we calculate $\tau=100 \mathrm{~s}$.

Maps generated following the stabilization of the temperature field (170 s) show the homogeneity of the sample deformation. At the bottom of the sample, the strain field is influenced by the specific mechanical boundary condition of the bottom, which is fixed without any horizontal or vertical displacement.

Results obtained in the laboratory, unlike in the simulation, show that permanent changes in microstructure occur with temperature. As the sample is heated for the first time, a high acoustic emission (AE) rate is contemporaneous with large-and mostly permanent-apparent reductions in velocity with temperature (Griffiths et al. 2018). P-wave velocity is reduced by $50 \%$ of the initial value at $450{ }^{\circ} \mathrm{C}$, and $40 \%$ upon cooling: the relative time shift is positive during heating and negative during cooling and a net apparent decrease in velocity of $17 \%$ is highlighted following the first cycle. Griffiths et al. (2018) observed fewer permanent changes in wave velocity during the second heating/cooling cycle, and less again during the third cycle. During these two cycles, the change in velocity was reversible: the velocity decreased by roughly $10 \%$ of its initial value when the sample was heated to $450{ }^{\circ} \mathrm{C}$ and increased again during cooling. Griffiths et al. (2018) attributed the reversible velocity increase/decrease to the elastic expansion/contraction of crystals, and the associated opening/closing of microcracks.

To illustrate this variability of behavior during the cycles of the experiments, the three panels of Fig. 7 show zooms of the waveforms, from 50 to $150 \mu \mathrm{s}$. The $100 \mu \mathrm{s}$ long waveforms recorded during the first, second and third heating of the sample are stacked. The horizontal axis indicates time in the coda, the vertical axis the sample temperature, the gray level the waveform amplitude. Figure 7 first illustrates the "stretching" effect, showing that the arrival times of the scattered waves appear shifted towards later arrival times when the sample temperature rises. The waveform looks like physically stretched when the temperature increases (in opposition to the compression of the waveforms observed during cooling) and the CWI method applied intends to quantify the relative variation of time delays with time, $\varepsilon=\delta t / t$. The comparison between the three panels shows then that the amplitude of this stretching phenomenon is different between the first and

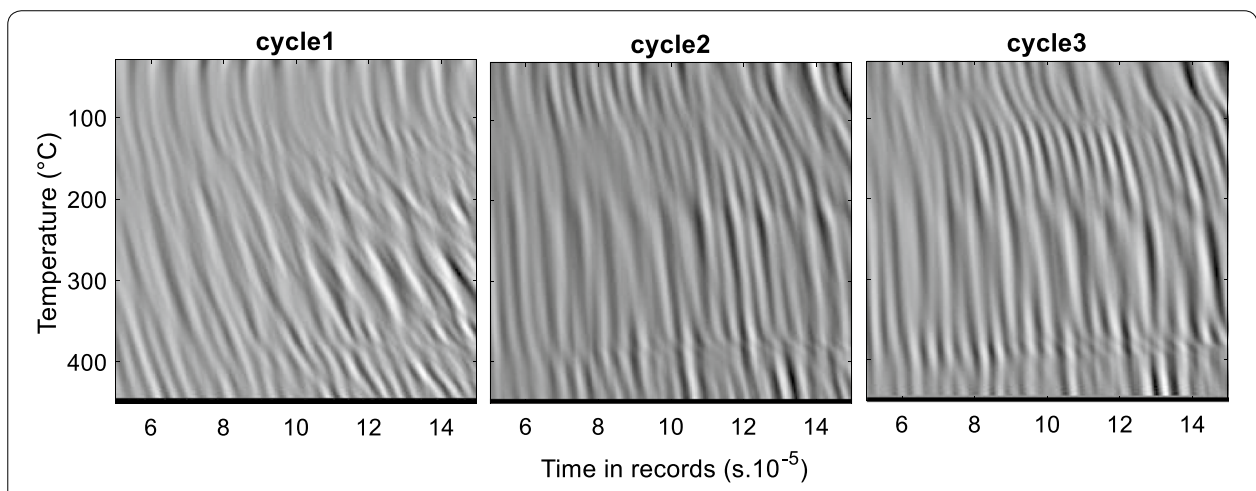

Fig. 7 Zoom within a $100 \mu$ s duration window (from 50 to $150 \mu \mathrm{s}$, after first arrivals) of the waveforms recorded in the laboratory during three heating cycles. Grayscale indicates the normalized amplitude of the particle motion 
subsequent cycles. Indeed, it shows that the amplitude of the relative time delay-with reference to the waveform recorded at ambient temperature-increases significantly from $180{ }^{\circ} \mathrm{C}$, which is not the case during the second and third cycles for which the stretching of the waveforms is more linear with temperature and of similar amplitude. Figure 7 highlights that the irreversible non-linear changes linked to thermal microcracking dominate during the first cycle (Griffiths et al. 2018), but are reduced during subsequent cycles, which are instead dominated by the thermo-elastic deformation of the sample. We compare in the following the simulated CWI measurements to those recorded during the second or third cycle in the laboratory such that the numerical model describes the physics of the experiment.

\section{CWI results}

In Fig. 8, we stacked the original waveforms recorded during three heating and cooling cycles, for both the laboratory measurements and the simulation. It enables us to compare the numerical response of the sample of the simulation to the behavior of the real granite sample for a similar thermal loading. Figure 8a shows the temporal evolution of the Westerly Granite sample temperature measured during the experiment. Figure $8 \mathrm{~b}$, c presents the $2 \mathrm{~ms}$ long waveforms recorded in the laboratory and in the simulation, respectively. The original waveforms recorded in the experiments are stacked every $5{ }^{\circ} \mathrm{C}$ and every $10{ }^{\circ} \mathrm{C}$ for the simulated recordings. During the first cycle, when large intrinsic variations linked to thermal microcracking dominate the experiments, the simulation results show distinct behavior. During the second and third cycles, the experiments and simulations have a similar linear behavior of relative travel time delay with temperature when thermo-elastic deformation dominates. During these cycles, the acoustic and mechanical behavior of the granitic sample is comparable to that of the sample in the

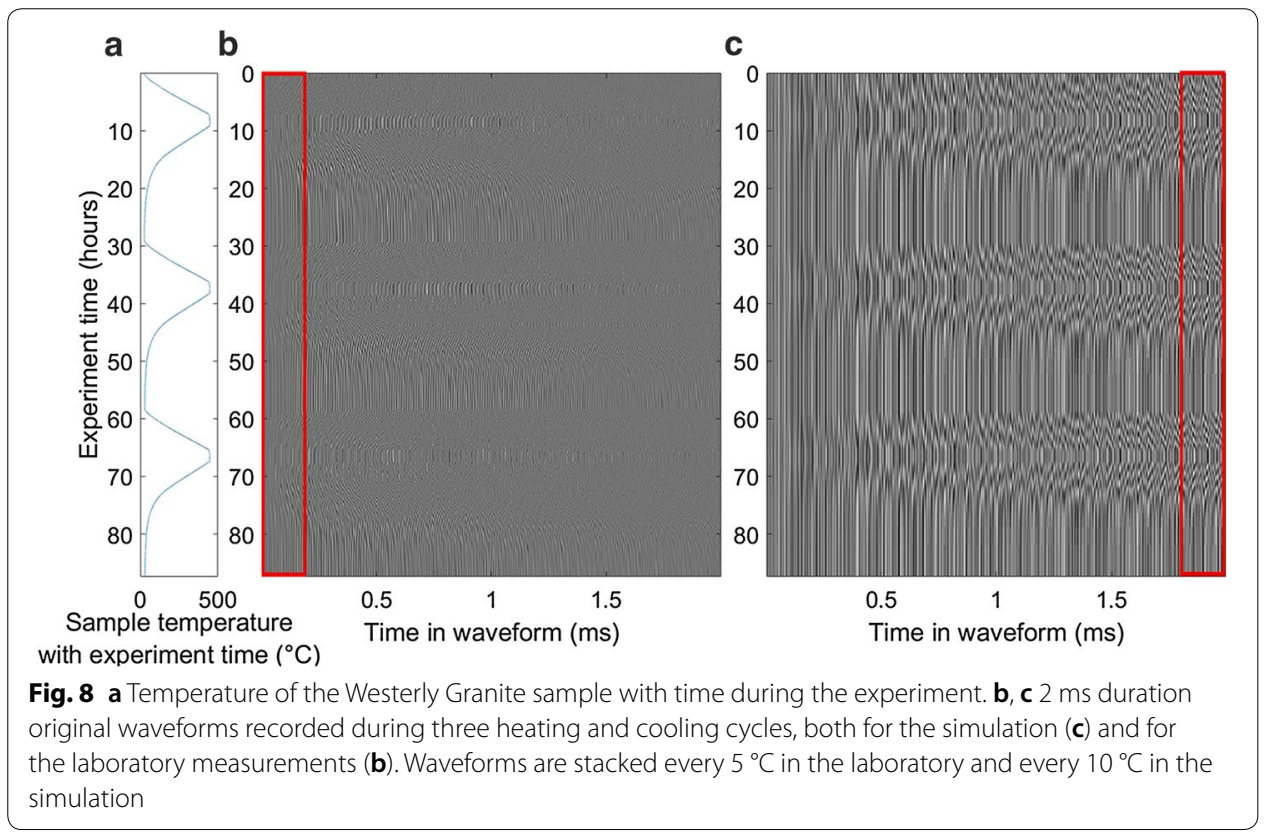


simulation, which allows us to compare the CWI measurements from Griffiths et al. (2018) to the simulated ones.

We zoom in on $100 \mu$ s duration windows within the laboratory recordings (Fig. 9a) and in the simulated recordings (Fig. 9b) to visually compare the measurements, with a specific focus on the second heating cycle. The windows selected in the numerical data of Fig. 8 are centered late within the coda (around $1.75 \mathrm{~ms}$ ) and early for the laboratory records (around $0.1 \mathrm{~ms}$ ). While the experiments and simulations exhibit similar behavior in terms of time shift, the diffusion of the wavefield due to wave scattering/reflections within the numerical medium is less pronounced than it is in the experimental granite sample: the waveform changes highlighted in each panel of Fig. 9 with heating or cooling show differences between the simulation and the experiment. We expect the grain inclusions in the laboratory granite sample also contribute to diffuse the wavefield, while multiple reflections diffuse the wavefield in the numerical sample considered in the simulation. The comparison of linearized energy density functions led (Fig. 4) already showed that a longer time is required to obtain a diffuse field in the medium of the simulation than in the real granite sample. It is thus necessary to consider that the effect of the thermal loading on the propagated wavefield is more pronounced in the real granite sample than in the simulated medium to exhibit a similar behavior in the selected windows in Fig. 9. The records are compared here visually and we present quantified measurements in the following paragraphs.

The impact of thermal deformation on the wave diffusion was quantified from CWI measurements following both an experimental and a numerical approach. If Figs. 8 and 9 illustrate the similarity in behavior of the CWI during the simulation and the second and third heating cycles in the laboratory, Fig. 10 gives quantified evidence for the similarity in behavior: the laboratory and the simulated CWI results show both a quasi-linear variation of relative time shift $\varepsilon$ with temperature. We recall that wave velocities are constant in this numerical approach, and that the simulation focuses on the consequences of the

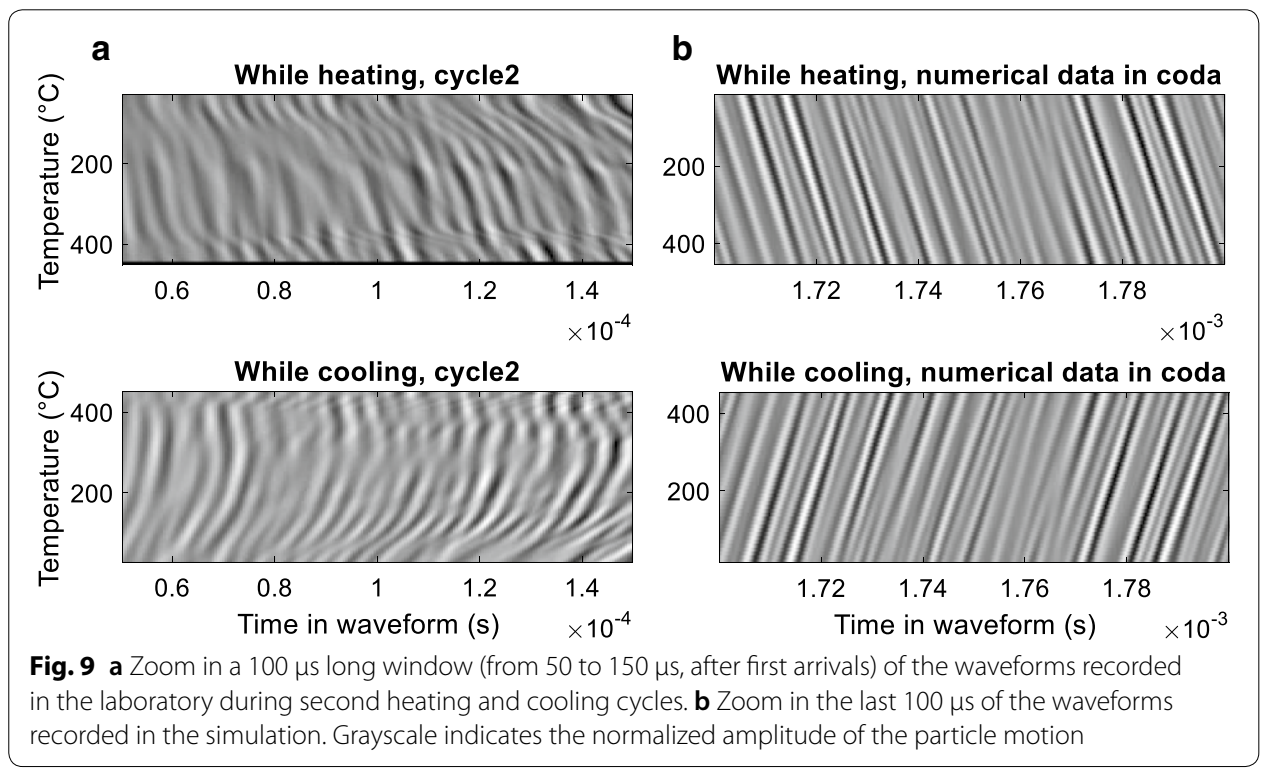




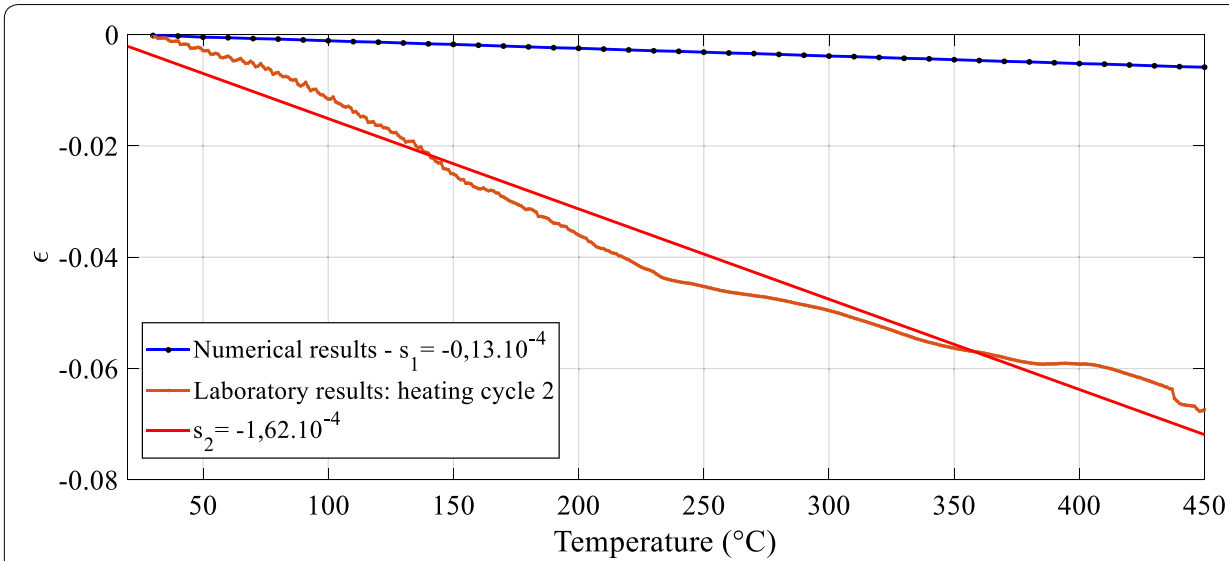

Fig. 10 Comparison of simulated and laboratory CWI measurements. We represent the CWI measurements obtained in the laboratory (orange line) during the second heating cycle of Westerly Granite and in the simulation (blue line) with temperature measured at the middle of the sample. Relative time delays are measured by the "stretching technique" when comparing the waveform recorded at a given temperature with respect to the "reference" waveform. A linear regression is fitted to the measurements to estimate the slopes $s_{\text {, }}$ and $s_{2}$ (blue line and red line for simulated and laboratory results, respectively)

thermo-elastic deformation of the sample. The simulated CWI results monitor the reversible and elastic deformation generated through thermal loading. Interestingly, the phenomenon modeled in our thermo-elastic simulation is shown to be dominant in the granite sample during the second cycle at the laboratory.

If the physics at play prove to be comparable, Fig. 10 also gives quantified arguments of the difference in amplitude between the behavior in the laboratory and in the simulation. Figure 10 shows the linear regressions Eq. (3) fitted to both CWI measurements. The slope $\mathrm{d} \varepsilon / \mathrm{d} T$ of the linear regression fitted to the numerical results, noted $s_{1}$, is one order of magnitude lower than slope $s_{2}$ estimated for laboratory results: $s_{1}$ (simulation) represents $8 \%$ of $s_{2}$ (laboratory). This comparison suggests that even if the behaviors are similar, the sensitivity to temperature is higher in the experiment than in the simulation.

\section{Discussion}

\section{Thermal expansion effect}

Interestingly, the isotropic thermal expansion of the medium is retrieved by the CWI results obtained during the simulation of the thermo-elastic deformation of the medium of the simulation, a homogeneous $2 \mathrm{D}$ rectangular sample (Fig. 8). Indeed, slope $s_{1}$ equals the isotropic thermal expansion coefficient, which is an input for Code_Aster. The CWI signal measured in the simulation is likened to a deformation signal. To discuss the physical origin of the simulated CWI measurements, we propose to compare these results to those expected due to the medium density changes. In the simulation of the thermo-elastic deformation, density, Young's and bulk modulus and thermal expansion are constant with temperature. The mesh grid is the only evolving parameter during successive steps, which corresponds to constant wave speeds $v_{\mathrm{P}}$ and $v_{\mathrm{S}}$. In a model considering only the effect due to the medium density changes, we show that the relative variation of travel time is related to the volumetric strain by a linear relation with a -0.5 coefficient of proportionality. 
Indeed, dilatation will result in a velocity change, according to Eq. (3). Differentiating Eq. (3) leads to the following relation:

$$
\delta v / \nu_{0}=1 / 2 \cdot \delta E / E-1 / 2 \cdot \delta \rho / \rho_{0} .
$$

With a constant bulk modulus $E$ and a constant mass, with the volume of the sample Vol, we expect the following relation between wave velocity changes and volumetric changes:

$$
\delta v / v_{0}=1 / 2 \cdot \delta \mathrm{Vol} / \mathrm{Vol}_{0}
$$

If we assume an infinitesimal deformation of the sample, we expect the relative variation of volume to equal the volumetric strain $e_{\mathrm{vol}}$, such that Eq. (3) yields the linear relation between relative variation of travel time and volumetric deformation with a -0.5 coefficient of proportionality:

$$
\delta t / t=-1 / 2 \cdot e_{\mathrm{vol}} \text {. }
$$

In our simulation, the volumetric strain is estimated from Eq. (13). With the volumetric thermal expansion coefficient $\alpha_{v}$, a change in temperature $\delta T$ results in a volumetric deformation:

$$
e_{\mathrm{vol}}=\delta \mathrm{Vol} / \mathrm{Vol}_{0}=\alpha_{v} \cdot \delta T \text {. }
$$

Equation (13) is obtained by integration of the following relation, assuming that the thermal expansion coefficient is isotropic and constant with temperature, and that the volume changes are small compared to the original volume:

$$
e_{\mathrm{vol}}=\delta \mathrm{Vol} / \mathrm{Vol}_{0}=\exp \left(\int_{T_{i}}^{T_{f}} \alpha_{\nu}(T) \mathrm{d} T\right)-1
$$

The thermal expansion coefficient equals in our 2D simulation and in the 3D laboratory experiments, respectively, twice and three times the linear isotropic expansion coefficient, inputted in Code_Aster. In Fig. 11, the relative time delay $\varepsilon$ measured in the laboratory and in the simulation are represented as a function of volumetric strain. The linear regression fitted to the experimental results with a slope of -0.55 , is consistently superimposed on the relative time shifts calculated from Eq. (12) related to the medium density changes. It shows that the CWI signal recorded in the simulation monitors the sample dilatation, supposedly homogeneous.

\section{Temperature effects on elastic moduli}

The difference in the slopes of the linear regression fitted to the simulated and laboratory CWI results (see Figs. 8, 11), suggest that another mechanism is at play. Microcrack widening might also occur during second and third cycles. The deformation calculated from the thermal expansion coefficient does not explain on its own the larger time shifts measured in the laboratory experiment in the absence of microcracking (i.e., during cycles 2 and 3). Up to now, in the thermo-elastic deformation simulation, we assumed that the density and the bulk and Young's modulus are independent of temperature. However, several measurements show that it is a strong approximation. For example, 


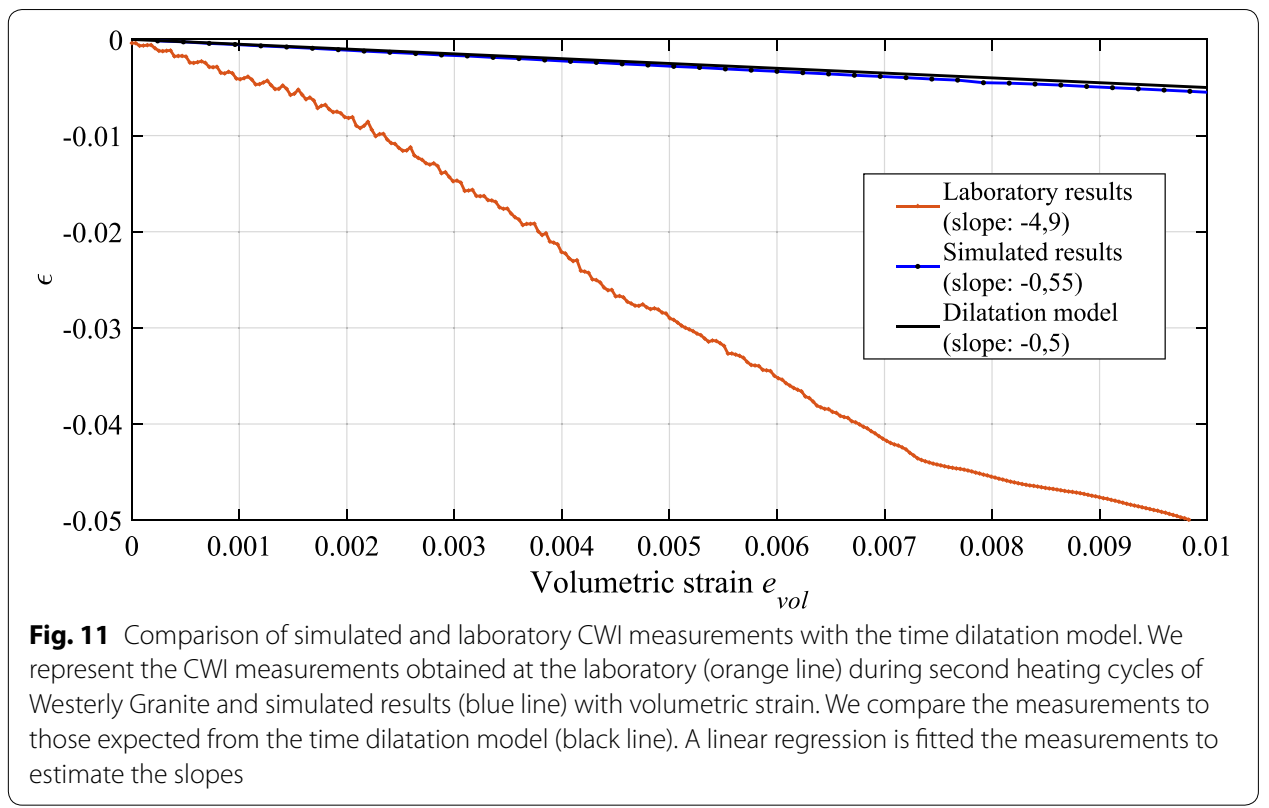

Heap and Faulkner (2008) showed that the Young's modulus of Westerly Granite can decrease significantly with increasing microcrack damage. Heard and Page (1982) show an important effect on bulk modulus $K$ and Young's modulus $E$ for Westerly Granite when temperature is increased to $300{ }^{\circ} \mathrm{C}$. Heard and Page (1982) give measurements of the relative variation of Young's modulus $E / E_{0}$ and of bulk modulus $K / K_{0}$ at different confining pressures, where the reference values $E_{0}$ and $K_{0}$ are measured for maximum confining pressure. For 7.6 $\mathrm{MPa}$-the lowest confining pressure-the ratio $E / E_{0}$ varies from 0.5 to 0.25 when the sample is heated up to $300{ }^{\circ} \mathrm{C}$. For a confining pressure of 13.8-27.6 MPa, the ratio $K / K_{0}$ also varies from 0.45 to 0.15 in this range of temperature. As these parameters are related to wave velocities, we can expect a significant effect on the wave velocities when a sample is heated up to $450^{\circ} \mathrm{C}$, and independently of the density effects discussed in previous paragraph.

We propose to use the measurements of Heard and Page (1982) to investigate the effect of the temperature dependency of the mechanical parameters on the wave velocities. We used seven measurements of normalized Young's and bulk modulus $E / E_{0}$ and $K /$ $K_{0}$ reported in Heard and Page (1982) for temperatures ranging from ambient temperature $\left(20^{\circ} \mathrm{C}\right)$ to $300{ }^{\circ} \mathrm{C}$, for an intact Westerly Granite sample. We calculated the relative variation of $\mathrm{P}$ and $\mathrm{S}$-wave speed from their proposed dependency with temperature of the density $\rho$, the bulk modulus $K$ and the Young's modulus $E$ using Eqs. (15) and (16):

$$
\begin{aligned}
& v_{\mathrm{P}}=\sqrt{\frac{3 \cdot K \cdot(3 \cdot K+E)}{\rho \cdot(9 \cdot K-E)} .} \\
& v_{\mathrm{S}}=\sqrt{\frac{3 \cdot K \cdot E}{\rho \cdot(9 \cdot K-E)}} .
\end{aligned}
$$


Normalized Young's moduli $E / E_{0}$ are chosen at the lowest confining pressure (7.6 MPa). Normalized bulk moduli $K / K_{0}$ at temperatures ranging from 150 to $300{ }^{\circ} \mathrm{C}$ are also chosen at 7.6 MPa. We inferred the values at lower temperatures $\left(20-150^{\circ} \mathrm{C}\right)$ from the measurements obtained at a higher confining pressure (13 MPa), because the measurements were not collected at $7.6 \mathrm{MPa}$ in this temperature range. The temperature dependency of the density is obtained from Eq. (17), where the volumetric coefficient of thermal expansion $\alpha_{v}$ is constant with temperature:

$$
\rho(T)=\rho\left(T_{0}\right) /\left(1+\alpha_{\nu} \cdot \delta T\right) .
$$

In Fig. 12, we compared the relative variation of $\mathrm{P}$ and $\mathrm{S}$-wave velocities calculated from Eqs. (15) and (16) to the relative variation of P-wave velocities calculated from the first arrival times measured in the laboratory for the Westerly Granite sample during the three repeated cycles (Griffiths et al. 2018). In Fig. 12, we represent the relative wave velocity variations with temperature obtained either from a direct measurement of Griffiths et al. (2018) (dashed lines) or from an indirect computation using the measurements of density and bulk and Young's moduli from Heard and Page (1982) (colored lines). The comparison aims to evaluate the relevance of including the dependence with temperature of the mechanical parameters into the simulation. The large relative velocity variations deduced from the temperature dependence of the bulk and Young's moduli with temperature from Heard and Page (1982) are closer in amplitude and in temperature gradient to the measurements during the first cycle of Griffiths et al. (2018). Importantly, relative bulk moduli and Young's moduli used in the calculations are obtained from measurements on an intact Westerly Granite sample, which corresponds to the case of the first heating cycle in the laboratory experiments of Griffiths et al. (2018). It is different for the second and third cycles, previously used as a reference when comparing CWI results, as irreversible changes in wave velocities were already observed during

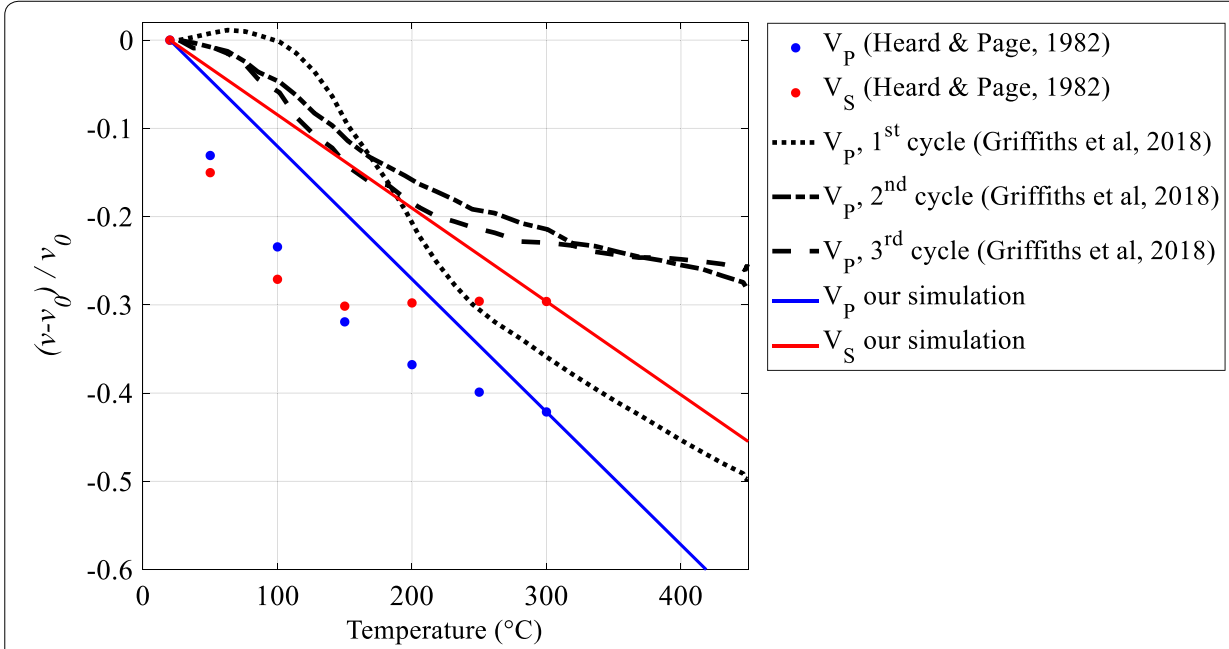

Fig. 12 Relative variation of P-wave speed measured in the laboratory during cycles 1-3 (black line), as a function of the temperature on Westerly Granite (Griffiths et al. 2018). The blue and red dots represent, respectively, the relative variation $\mathrm{P}$-wave speed and for $\mathrm{S}$-wave speed deduced from the $E(T), K(T)$ and density measurements of (Heard and Page 1982) also for Westerly Granite. The blue and red lines represent, respectively, the relative variation P-wave speed and for S-wave speed introduced in our simulation to take into account the experimental measurements of (Heard and Page 1982) 
the previous cycle and linked to microcracking. This dependence with temperature of the mechanical parameters is expected to explain part of the difference in behaviors, as reported in Fig. 12.

To test the influence of the dependence in temperature of the elastic parameters $\rho(T), E(T)$ and $K(T)$, we performed complementary simulations, where the temperature dependence is introduced. We included the following laws in the simulation to take into account the experimental results of Heard and Page (1982), where $T$ is the temperature $\left({ }^{\circ} \mathrm{C}\right): v_{\mathrm{P}}(T)=-7.19 \cdot T+4.92 \cdot 10^{3}$ and $v_{\mathrm{S}}(\mathrm{T})=-2.79 \cdot \mathrm{T}+2.70 \cdot 10^{3}$. Figure 12 includes the relative variation of $\mathrm{P}$ and $\mathrm{S}$-wave velocities associated to these laws (colored lines) to show the link between the experimental measurements of Heard and Page (1982) and the dependence included in the simulation. The temperature dependence of density is obtained from Eq. (17).

Figure 13 shows that the decrease in wave speed with increasing temperature results in the stretching of the waveforms recorded in the simulation. The delays measured between the "reference" and "perturbed" waveforms at a given temperature (for example $T=100{ }^{\circ} \mathrm{C}$ in Fig. 13) are higher when we include the temperature dependence of the mechanical parameters. Figure 13a shows that differences are present between the first $0.1 \mathrm{~ms}$ of the "reference" and "perturbed" waveform only when the effect of the temperature on the modules is included in the simulation. In Fig. 13b, a difference is already highlighted between the "reference" and "perturbed" without temperature dependence of the moduli, but it is exacerbated with the introduction of the $E(T), K(T)$ and $\rho(T)$ laws in the simulation. It results in an increase of the relative time shifts $\varepsilon$ measured with reference to the waveform recorded at ambient temperature $\left(20^{\circ} \mathrm{C}\right)$.

Figure 14 compares the CWI measurements obtained at the laboratory during the first cycle for an intact sample (red line) with those simulated with (black line) and without (blue line) the introduction of the temperature dependence of the mechanical parameters. The CWI results obtained in the experiments (red line) are better modeled by

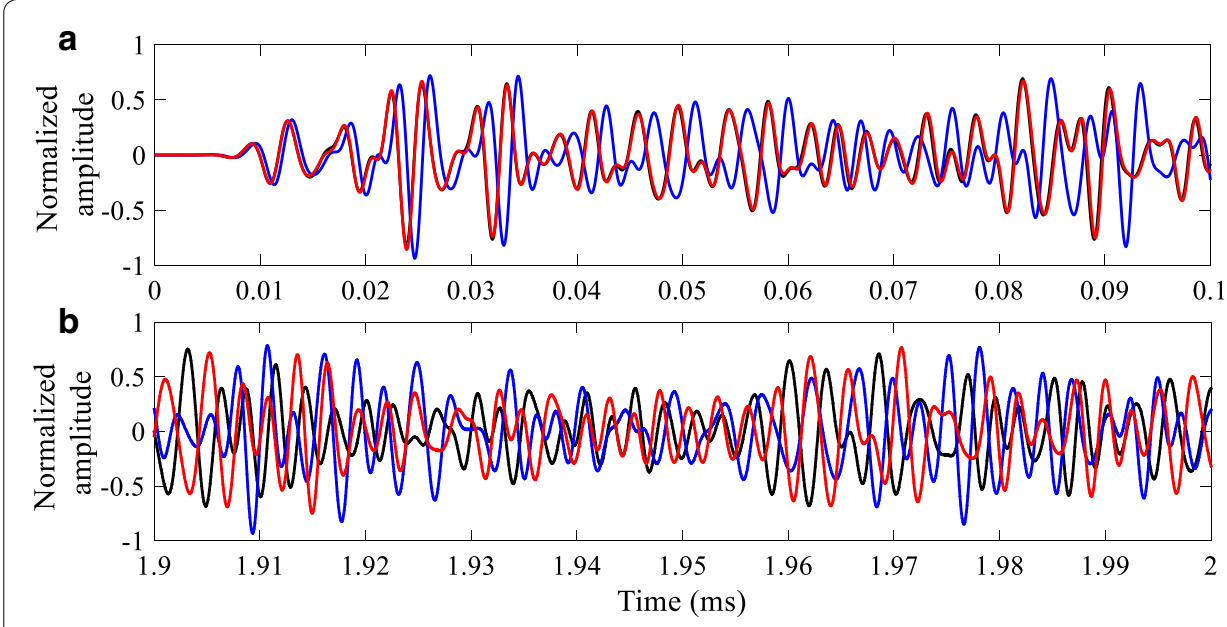

Fig. 13 Black line is the reference waveform recorded at ambient temperature $\left(20^{\circ} \mathrm{C}\right)$, red line is a waveform recorded at $100^{\circ} \mathrm{C}$ without introduction of the $E(T)$ and $K(T)$ laws in the simulation and blue line is a waveform recorded at $100^{\circ} \mathrm{C}$ after introduction of the $E(T)$ and $K(T)$ laws in the simulation a first $0.1 \mathrm{~ms}$ of the simulated waveforms $\mathbf{b}$ last $0.1 \mathrm{~ms}$ of the simulated waveforms 


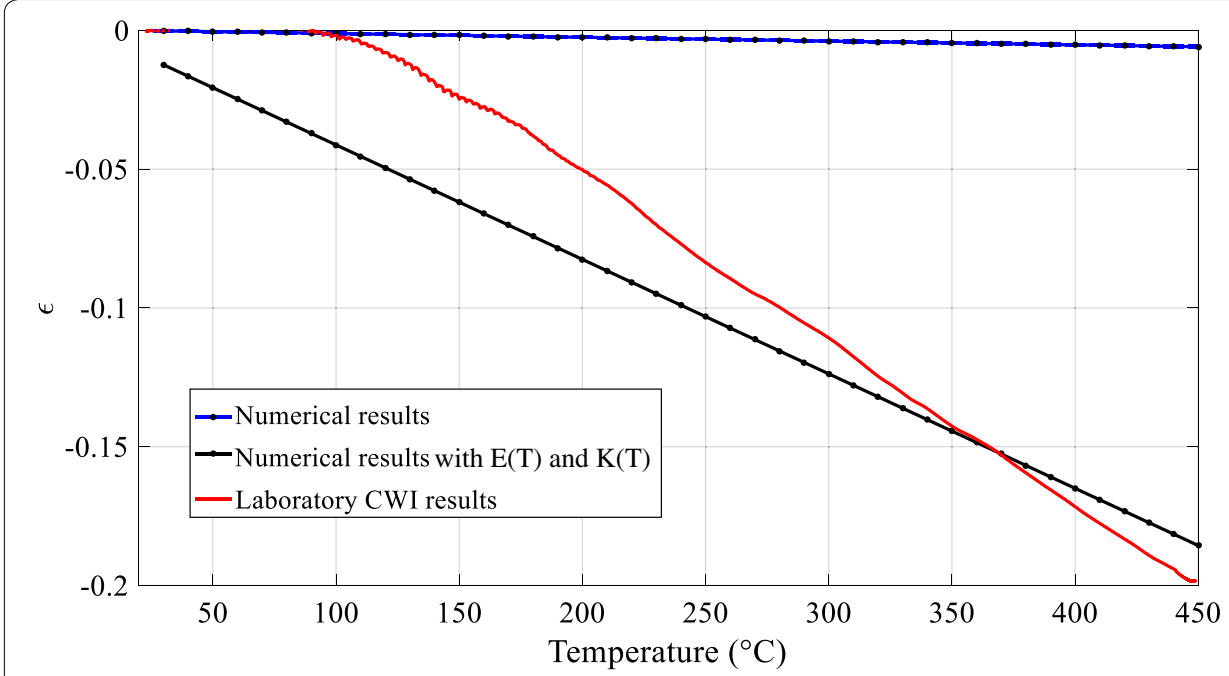

Fig. 14 Comparison of laboratory CWI measurements during the first cycle (red line) with the simulated CWI measurements obtained without (blue line) and with (black line) introduction of a dependency with temperature of the bulk modulus $K$ and of the Young's modulus $E$. Relative time delays $\varepsilon$ are calculated from the stretching technique. A linear regression is fitted to the measurements. The slope of the linear regression fitted to the simulated CWI results is $s_{1}=-0.13 \cdot 10^{-4}$ without introduction of the temperature dependency and it is $-4.1 \cdot 10^{-4}$ when the dependency is added to the simulation

the complementary simulation (black line) than without addition of the temperature dependence of $E$ and $K$. The CWI measurements overestimate the experimental results obtained during cycle two and three, because they include partial thermal damage microcracking that are less important in the experimental results chosen for comparison in "CWI results". During the first cycle, the intact sample is subject to large velocities perturbations, which are retrieved in the simulation from the time dependence of the density, Young's modulus and bulk modulus. This result and the difference between the simulations and the experiments in cycles 2 and 3 indicate still a softening of the sample during each heating cycle, unexplained by deformation alone.

\section{Geothermal implications}

In the context of geotechnical activities, it is of primary interest to understand the physical and mechanical properties of the rock mass stimulated by human activities such as mining, hydraulic fracturing, oil and gas production, waste and $\mathrm{CO}_{2}$ storage, and heat and water extraction or injection. For geothermal applications this monitoring is crucial as large-volume fluid injections and extractions can produce changes in the local stress field resulting in triggered instabilities which may increase the seismic risk in regions of little natural seismicity (e.g., Ellsworth 2013). Today, most monitoring methods used to study reservoir behavior are based on microseismicity (Shapiro 2008). Induced seismicity can indeed provide estimates of rock properties following hydraulic stimulations and can be used to assess the stress field and the resulting seismic hazard (e.g., Shapiro et al. 2007; Bachmann et al. 2011). However, such methods cannot be used as efficiently when the reservoir has matured and as the induced seismicity drops significantly when the fractured percolation networks are established (Schoenball et al. 2014). For a few years, CWI has provided a new tool to monitor the time evolution of geological 
structures such as mud landslides, fault zones, the water table, etc. (e.g., Mainsant et al. 2012; Brenguier et al. 2008; Hillers et al. 2015a; Lecocq et al. 2017). When applied on coda waves reconstructed from ambient seismic noise, CWI allows us to monitor active structures without the use of microseismicity, which opens perspectives for its application to monitor geothermal activities in several locations such as St. Gallen, Switzerland (Obermann et al. 2015), Basel, Switzerland (Hillers et al. 2015b) and Rittershoffen, France (Lehujeur et al. 2014, 2017), where a deep geothermal plant $(2500 \mathrm{~m})$ is installed (Baujard et al. 2017). CWI, therefore, emerges as a powerful tool to monitor geothermal reservoirs and could help guide stimulation strategies designed to boost reservoir productivity.

The work presented in this study will assist the interpretations of CWI at existing and future geothermal sites within the Upper Rhine Graben. Seismic interferometry studies generally assume that velocity changes dominate the CWI signals such that they commonly neglect the contribution of deformation on the signal. The specificity of our thermo-elastic deformation simulation is the constant wave velocity and the interpretation of the relative time shifts as a deformation signal induced by the thermal dilatation of the sample. The addition of the temperature dependence of the mechanical parameters enables us to model large wave velocity variations induced by the temperature dependency. The measured relative time shifts $\varepsilon$ could be interpreted in the field by slight changes in the stress state in a reservoir rock mass due to thermal fluctuations, which opens perspectives for potential detection by these methods of stress changes during stimulation or exploitation.

The combination of laboratory experiments with numerical simulations also allows to us discuss the relative influences of the physical properties at the origins of the delays measured in CWI. Laboratory measurements embrace all the distinctive contributions to the signal (i.e., changes in properties such as velocity, attenuation, anisotropy, and scattering properties). The comparison of CWI measurements obtained in the simulation with those computed for real laboratory experiments opens real perspectives to distinguish the physical processes of the measured time shifts. Such a comparison is applied here to the cycle heating of Westerly Granite, which is a relevant context when dealing with thermally dynamic environments such as geothermal reservoirs. If the simulation isolates the reversible elastic response of the sample to the thermal loading, the difference in CWI results suggest that there are other reversible deformation mechanisms at play in the laboratory experiments. This comparison of results highlights the partitioning between the different physical processes responsible for time delay in CWI measurements. The discrimination between the different effects induced by thermal variations-changes of properties, such as velocity, attenuation, anisotropy, and scattering properties-of the delays measured with CWI type of signals remains challenging. However, the $8 \%$ ratio highlighted in our study when comparing the slopes $\mathrm{d} / \mathrm{d} T$ of the linear regression fitted to simulated and laboratory CWI results, provides a first estimate of the thermal strain contribution to CWI measurements. Therefore, the interferometry technique could potentially detect stress changes linked to a reservoir thermal variation during stimulation or exploitation which could help to guide stimulation strategies designed to optimize reservoir productivity. 


\section{Conclusions}

This study consists of a comparison of CWI results obtained from two recently developed approaches and aims at monitoring changes within a dispersive Westerly Granite sample heated up to $450{ }^{\circ} \mathrm{C}$. The first approach is a laboratory experiment, in which the granite sample is submitted to three successive heating/cooling cycles (Griffiths et al. 2018). Griffiths et al. (2018) highlighted strong irreversible changes during the first cycle and reversible changes in second and third cycles. This laboratory experiment is duplicated in a numerical scheme developed by Azzola et al. (2018) that aims to explain part of the CWI signal measured in the experiments. An important simplification of the numerical modeling is the definition of a homogeneous sample with representative elastic moduli and thermal expansion coefficient but where scattering is only obtained from multi-reflections on the boundaries. Interestingly, such a numerical approach shows the specific effect of the thermo-elastic deformation of the sample, while all the distinctive contributions to the experimental CWI signal are embraced at once in the experiment. In the simulation, when we assume that the coefficient of thermal expansion, the bulk modulus and the Young's modulus are constant with temperature, the two main results are as follows:

1. The isotropic thermal expansion of the medium is retrieved from the relative variation of delay $\varepsilon=t / t$ measured in the simulation with temperature $T$. CWI results obtained from the simulation in a homogeneous $2 \mathrm{D}$ rectangular sample consist of a deformation signal, due to the elastic and homogeneous deformation of the sample.

2. Comparison with the CWI signal measured under comparable conditions at the laboratory shows that the relative variation of delays $\varepsilon$ measured in the simulation are significantly lower (12 times) than in the simulated experiments, as depicted by the slopes $\mathrm{d} / \mathrm{d} T$ of the linear regression fitted to simulated and laboratory CWI results, noted, respectively, $s_{1}$ and $s_{2}$ (Fig. 10). In the experiments, and in the absence of microcracking (i.e., during cycles 2 and 3 ), deformation alone only partly explains the impact of temperature changes on CWI measurements.

To discuss the highlighted difference, we propose to additionally model the temperature dependence of the elastic moduli. Complementary simulations explain the higher magnitude for the relative variation of delays measured in the experiments when adding the temperature dependency of the linear thermal expansion coefficient $\alpha$, of the bulk modulus and of the Young's modulus (Heard and Page 1982). This work opens a potential use of CWI for the monitoring of thermal changes in deep geothermal reservoirs.

Authors' contributions

JA performed the modeling, under the guidance of JS, DZ, VM, and FM. LG performed the laboratory experiment, under the supervision of $\mathrm{MJH}, \mathrm{PB}$, and JS. JA wrote the manuscript, with help from all the co-authors. All authors read and approved the final manuscript.

\section{Author details}

${ }^{1}$ Institut de Physique du Globe de Strasbourg, Université de Strasbourg/EOST, Strasbourg, France. ${ }^{2}$ Petroleumsgeomekanikk og geofysikk, NGI (Norwegian Geotechnical Institute), Oslo, Norway. ${ }^{3}$ ICUBE, Illkirch, France. 


\section{Acknowledgements}

The authors would like to thank Bertrand Renaudié for sample preparation and Alexandre Schubnel for kindly furnishing the block of Westerly Granite (with the help of Lucas Pimienta), and Alain Steyer and Laurent Rihouey for their help with the construction of the experimental setup. We acknowledge in particular the important contribution of Olivier Lengliné in the setup of the experimental device and in the interpretation of the experimental data. For the use of SPECFEM2D, a free software downloadable at https://github.com/geodynamics/specfem2d.git, we thank the Computational Infrastructure for Geodynamics (http://geodynamics.org) which is funded by the National Science Foundation under awards EAR-0949446 and EAR-1550901. For the use of Code_Aster, which is also a free software downloadable at https://www. code-aster.org/V2/spip.php?rubrique7, we thank Electricité de France (EDF).

\section{Competing interests}

The authors declare that they have no competing interests.

Availability of data and materials

The DOl of the data presented here is https://doi.org/10.6084/m9.figshare.6226874.

\section{Funding}

This work has been published under the framework of the LABEX ANR-11-LABX-0050-G-EAU-THERMIE-PROFONDE and benefits from a funding from the state managed by the French National Research Agency (ANR) as part of the'Investments for the Future' program. We acknowledge ANR grant CANTARE (ANR-15-CE06-0014-01). It has also been funded by the EU projects DESTRESS (EU H2020 research and innovation program, grant agreement No. 691728).

\section{Publisher's Note}

Springer Nature remains neutral with regard to jurisdictional claims in published maps and institutional affiliations.

Received: 15 May 2018 Accepted: 5 October 2018

Published online: 25 October 2018

\section{References}

Aichholzer C, Duringer P, Orciani S, Genter A. New stratigraphic interpretation of the Soultz-sous-Forêts 30-year-old geothermal wells calibrated on the recent one from Rittershoffen (Upper Rhine Graben, France). Geotherm Energy. 2016;4:13. https://doi.org/10.1186/s40517-016-0055-7.

Azzola J, Schmittbuhl J, Zigone D, Magnenet V, Masson F. Direct modeling of the mechanical strain influence on coda wave interferometry. J Geophys Res Solid Earth. 2018;123:3160-77. https://doi.org/10.1002/2017JB015162.

Bachmann CE, Wiemer S, Woessner J, Hainzl S. Statistical analysis of the induced Basel 2006 earthquake sequence: introducing a probability-based monitoring approach for enhanced geothermal systems. Geophys J Int. 2011;186:793807. https://doi.org/10.1111/j.1365-246X.2011.05068.x.

Baujard C, Genter A, Dalmais E, Maurer V, Hehn R, Rosillette R, Vidal J, Schmittbuhl J. Hydrothermal characterization of wells GRT-1 and GRT-2 in Rittershoffen, France: implications on the understanding of natural flow systems in the rhine graben. Geothermics. 2017;65:255-68. https://doi.org/10.1016/j.geothermics.2016.11.001.

Brace WF, Paulding BW, Scholz C. Dilatancy in the fracture of crystalline rocks. J Geophys Res. 1966;71:3939-53. https:// doi.org/10.1029/JZ071i016p03939.

Brace WF, Walsh JB, Frangos WT. Permeability of granite under high pressure. J Geophys Res. 1968;73:2225-36. https://doi. org/10.1029/JB073i006p02225.

Brenguier F, Campillo M, Hadziioannou C, Shapiro N, Nadeau RM, Larose E. Postseismic relaxation along the San Andreas fault at Parkfield from continuous seismological observations. Science. 2008:321(5895):1478-81.

Campillo M, Roux P. Crust and lithospheric structure-seismic imaging and monitoring with ambient noise correlations. 2015.

Chaki S, Takarli M, Agbodjan WP. Influence of thermal damage on physical properties of a granite rock: porosity, permeability and ultrasonic wave evolutions. Constr Build Mater. 2008;22:1456-61. https://doi.org/10.1016/j.conbuildma t.2007.04.002.

Dainty AM, Toksoz MN. Elastic wave propagation in a highly scattering medium—a diffusion approach. J Geophys Z Geophys. 1977;43:375-88.

Dainty AM, Toksöz MN. Seismic codas on the earth and the moon: a comparison. Phys Earth Planet Interior. 1981;26:25060. https://doi.org/10.1016/0031-9201(81)90029-7.

Darot M, Reuschlé T. Acoustic wave velocity and permeability evolution during pressure cycles on a thermally cracked granite. Int J Rock Mech Min Sci. 2000;37:1019-26. https://doi.org/10.1016/S1365-1609(00)00034-4.

David C, Menéndez B, Darot M. Influence of stress-induced and thermal cracking on physical properties and microstructure of La Peyratte granite. Int J Rock Mech Min Sci. 1999;36:433-48. https://doi.org/10.1016/S0148-9062(99)00010 $-8$

Dezayes C, Valley B, Maqua E, Syren G, Genter A. Natural fracture system of the Soultz granite based on UBI data in the GPK3 and GPK4 wells. In: Proceedings of the EHDRA scientific conference. Soultz-sous-Forêts France; 2005. p. 9.

Dwivedi RD, Goel RK, Prasad VVR, Sinha A. Thermo-mechanical properties of Indian and other granites. Int J Rock Mech Min Sci 2008;45(3):303-15. https://doi.org/10.1016/j.jijmms.2007.05.008.

Ellsworth WL. Injection-induced earthquakes. Science. 2013;341:6142. https://doi.org/10.1126/science.1225942.

Fredrich JT, Wong T. Micromechanics of thermally induced cracking in three crustal rocks. J Geophys Res Solid Earth. 1986;91:12743-64. https://doi.org/10.1029/JB091iB12p12743.

Genter A, Traineau H. Analysis of macroscopic fractures in granite in the HDR geothermal well EPS-1, Soultz-sous-Fore^ts, France. J Volcanol Geotherm Res. 1996;72:121-41. https://doi.org/10.1016/0377-0273(95)00070-4. 
Gérard A, Kappelmeyer O. The Soultz-sous-Forets project. Geothermics. 1987;16:393-9. https://doi.org/10.1016/03756505(87)90018-6.

Gérard A, Genter A, Kohl T, Lutz P, Rose P, Rummel F. The deep EGS (enhanced geothermal system) project at Soultz-sousForêts (Alsace, France). Pergamon. 2006.

Géraud Y. Variations of connected porosity and inferred permeability in a thermally cracked granite. Geophys Res Lett. 1994;21:979-82. https://doi.org/10.1029/94GL00642.

Grêt A, Snieder R, Scales J. Time-lapse monitoring of rock properties with coda wave interferometry. J Geophys Res Solid Earth. 2006;111(3):1-11. https://doi.org/10.1029/2004JB003354.

Griffiths L, Heap MJ, Wang F, Daval D, Gilg HA, Baud P, Schmittbuhl J, Genter A. Geothermal implications for fracture-filling hydrothermal precipitation. Geothermics. 2016;64:235-45. https://doi.org/10.1016/j.geothermics.2016.06.006.

Griffiths L, Heap MJ, Baud P, Schmittbuhl J. Quantification of microcrack characteristics and implications for stiffness and strength of granite. Int J Rock Mech Min Sci. 2017;100:138-50. https://doi.org/10.1016/j.jirmms.2017.10.013.

Griffiths L, Lengliné O, Heap MJ, Baud P, Schmittbuhl J. Thermal cracking in westerly granite monitored using direct wave velocity, coda wave interferometry, and acoustic emissions. J Geophys Res Solid Earth. 2018;123(3):2246-61. https:// doi.org/10.1002/2017JB015191.

Heap MJ, Faulkner DR. Quantifying the evolution of static elastic properties as crystalline rock approaches failure. Int J Rock Mech Min Sci. 2008;45:564-73. https://doi.org/10.1016/j.jirmms.2007.07.018.

Heap MJ, Kushnir AR, Gilg HA, Wadsworth FB, Reuschlé T, Baud P. Microstructural and petrophysical properties of the Permo-Triassic sandstones (Buntsandstein) from the Soultz-sous-Forêts geothermal site (France). Geotherm Energy. 2017;5:26.

Heard HC, Page L. Elastic moduli, thermal expansion, and inferred permeability of two granites to $350^{\circ} \mathrm{C}$ and 55 megapascals. J Geophys Res. 1982;87:9340. https://doi.org/10.1029/JB087iB11 p09340.

Hennino R, Trégourès N, Shapiro NM, Margerin L, Campillo M, van Tiggelen BA, Weaver RL. Observation of equipartition of seismic waves. Phys Rev Lett. 2001;86(15):3447-50. https://doi.org/10.1103/PhysRevLett.86.3447.

Hillers G, Husen S, Obermann A, Planès T, Campillo M, Larose E. Noise based monitoring and imaging of aseismic transient deformations induced by the 2006 Basel reservoir stimulations. Geophysics. 2015a;80:4. https://doi. org/10.1190/GEO2014-0455.1.

Hillers G, Ben-Zion Y, Campillo M, Zigone D. Seasonal variations of seismic velocities in the San Jacinto Fault area observed with ambient seismic noise. Geophys J Int. 2015b;202:920-32. https://doi.org/10.1093/gji/ggv151.

Hooijkaas GR, Genter A, Dezayes C. Deep-seated geology of the granite intrusions at the Soultz EGS site based on data from 5 km-deep boreholes. Geothermics. 2006;35:484-506. https://doi.org/10.1016/j.geothermics.2006.03.003.

Huenges E, Ledru P. Geothermal energy systems: exploration, development, and utilization. New York: Wiley; 2011.

Komatitsch D, Vilotte J-P. The spectral element method: an efficient tool to simulate the seismic response of 2D and 3D geological structures. Bull Seismol Soc Am. 1998;88:368-92.

Kranz RL. Microcracks in rocks: a review. Tectonophysics. 1983;100:449-80. https://doi.org/10.1016/0040-1951(83)90198 $-1$.

Kushnir ARL, Heap MJ, Baud P. Assessing the role of fractures on the permeability of the Permo-Triassic sandstones at the Soultz-sous-Forêts (France) geothermal site. Geothermics. 2018a;74:181-9. https://doi.org/10.1016/j.geothermic s.2018.03.009

Kushnir ARL, Heap MJ, Baud P, Gilg HA, Reuschlé T, Lerouge C, Dezayes C, Duringer P. Characterizing the physical properties of rocks from the Paleozoic to Permo-Triassic transition in the Upper Rhine Graben. Geotherm Energy. 2018b;6:16. https://doi.org/10.1186/s40517-018-0103-6.

Larose E, Hall S. Monitoring stress related velocity variation in concrete with a $2 \times 10^{-5}$ relative resolution using diffuse ultrasound. J Acoust Soc Am. 2009;125:1853-6. https://doi.org/10.1121/1.3079771.

Lecocq T, Longuevergne L, Pedersen HA, Brenguier F, Stammler K. Monitoring ground water storage at mesoscale using seismic noise: 30 years of continuous observation and thermo-elastic and hydrological modeling. Sci Rep. 2017;7:14241. https://doi.org/10.1038/s41598-017-14468-9.

Ledésert B, Hebert R, Genter A, Bartier D, Clauer N, Grall C. Fractures, hydrothermal alterations and permeability in the Soultz Enhanced Geothermal System. Comptes Rendus Geosci. 2010;342:607-15.

Lehujeur M, Vergne J, Schmittbuhl J, Maggi A. Investigating a deep geothermal reservoir using ambient noise correlation. In: EGU general assembly conference abstracts. 2014.

Lehujeur M, Vergne J, Maggi A, Schmittbuhl J. Ambient noise tomography with non-uniform noise sources and low aperture networks: case study of deep geothermal reservoirs in northern Alsace, France. Geophys J Int. 2017;208:193210. https://doi.org/10.1093/gji/ggw373.

Lockner DA. A generalized law for brittle deformation of Westerly Granite. J Geophys Res Solid Earth. 1998;103:5107-23. https://doi.org/10.1029/97JB03211.

Lu S-M. A global review of enhanced geothermal system (EGS). Renew Sustain Energy Rev. 2018;81:2902-21.

Mainsant G, Larose E, Brönnimann C, Jongmans D, Michoud C, Jaboyedoff M. Ambient seismic noise monitoring of a clay landslide: toward failure prediction. J Geophys Res Earth Surf. 2012;117:n/a-n/a. https://doi.org/10.1029/2011 JF0021 59.

Maqsood A, Gul IH, Anisur-Rehman M. Thermal transport properties of granites in the temperature range 253-333 K. J Phys Appl Phys. 2004;37:1405-9. https://doi.org/10.1088/0022-3727/37/9/016.

Meredith PG, Main IG, Clint OC, Li L. On the threshold of flow in a tight natural rock. Geophys Res Lett. 2012;39:n/a-n/a. https://doi.org/10.1029/2011GL050649.

Nakamura Y. Seismic energy transmission in an intensively scattering environment. J Geophys Z Geophys. 1977:43:389-99.

Nasseri MHB, Schubnel A, Young RP. Coupled evolutions of fracture toughness and elastic wave velocities at high crack density in thermally treated Westerly Granite. Int J Rock Mech Min Sci. 2007;44:601-16. https://doi.org/10.1016/j. ijrmms.2006.09.008. 
Nasseri MHB, Schubnel A, Benson PM, Young RP. Common evolution of mechanical and transport properties in thermally cracked westerly granite at elevated hydrostatic pressure. Pure Appl Geophys. 2009;166:927-48. https://doi. org/10.1007/s00024-009-0485-2.

Obermann A, Kraft T, Larose E, Wiemer S. Potential of ambient seismic noise techniques to monitor the St. Gallen geothermal site (Switzerland). J Geophys Res. 2015. https://doi.org/10.1002/2014jb011817.

Olasolo P, Juárez MC, Morales MP, Liarte IA. Enhanced geothermal systems (EGS): a review. Renew Sustain Energy Rev. 2016;56:133-44

Olivier G, Brenguier F, Campillo M, Lynch R, Roux P. Body-wave reconstruction from ambient seismic noise correlations in an underground mine. Geophysics. 2015;80(3):KS11-25. https://doi.org/10.1190/geo2014-0299.1.

Park C, Synn JH, Shin HS, Cheon DS, Lim HD, Jeon SW. An experimental study on the thermal characteristics of rock at low temperatures. Int J Rock Mech Min Sci. 2004;41:367-8.

Planès T, Larose E. A review of ultrasonic Coda Wave Interferometry in concrete. Cem Concr Res. 2013;53:248-55. https:// doi.org/10.1016/..cemconres.2013.07.009.

Poupinet G, Ellsworth WL, Frechet J. Monitoring velocity variations in the crust using earthquake doublets: an application to the Calaveras Fault, California. J Geophys Res Solid Earth. 1984;89(B7):5719-31. https://doi.org/10.1029/JB089 iB07p05719.

Ratdomopurbo A, Poupinet G. Monitoring a temporal change of seismic velocity in a volcano: application to the 1992 eruption of Mt. Merapi (Indonesia). Geophys Res Lett. 1995;22(7):775-8. https://doi.org/10.1029/95GL00302.

Roberts PM, Phillips WS, Fehler MC. Development of the active doublet method for measuring small velocity and attenuation changes in solids. J Acoust Soc Am. 1992;91(6):3291-302. https://doi.org/10.1121/1.402864.

Ryzhik L, Papanicolaou G, Keller JB. Transport equations for elastic and other waves in random media. Wave Motion. 1996;24(4):327-70. https://doi.org/10.1016/S0165-2125(96)00021-2.

Schoenball M, Dorbath L, Gaucher E, Wellmann JF, Kohl T. Change of stress regime during geothermal reservoir stimulation. Geophys Res Lett. 2014;41:1163-70. https://doi.org/10.1002/2013GL058514.

Schön SJ. Thermal properties. In: Handbook of petroleum exploration and production. Amsterdam: Elsevier; 2011. p. 337-72. https://doi.org/10.1016/S1567-8032(11)08009-8.

Sens-Schönfelder C, Wegler U. Passive image interferometry and seasonal variations of seismic velocities at Merapi Volcano, Indonesia. Geophys Res Lett. 2006;33. https://doi.org/10.1029/2006GL027797.

Shapiro SA. Microseismicity: a tool for reservoir characterization. Houten: EAGE Publications bv; 2008.

Shapiro SA, Dinske C, Kummerow J. Probability of a given-magnitude earthquake induced by a fluid injection. Geophys Res Lett. 2007;34:L22314. https://doi.org/10.1029/2007GL031615.

Siratovich PA, Villeneuve MC, Cole JW, Kennedy BM, Bégué F. Saturated heating and quenching of three crustal rocks and implications for thermal stimulation of permeability in geothermal reservoirs. Int J Rock Mech Min Sci. 2015:80:26580. https://doi.org/10.1016/j.jijmms.2015.09.023.

Snieder R. Coda wave interferometry for estimating nonlinear behavior in seismic velocity. Science. 2002;295(5563):22535. https://doi.org/10.1126/science.1070015.

Snieder R. The theory of coda wave interferometry. Pure Appl Geophys. 2006;163:455-73. https://doi.org/10.1007/s0002 4-005-0026-6.

Somerton WH. Thermal properties and temperature-related behavior of rock/fluid systems. Amsterdam: Elsevier; 1992.

Stehly L, Campillo M, Shapiro NM. Traveltime measurements from noise correlation: stability and detection of instrumental time-shifts. Geophys J Int. 2007;171:223-30. https://doi.org/10.1111/j.1365-246X.2007.03492.x.

Thirumalai K, Demou SG. Thermal expansion behavior of intact and thermally fractured mine rocks. Reston: ASCE; 1974. p. 60-71. https://doi.org/10.1063/1.2945937.

Villeneuve MC, Heap MJ, Kushnir AR, Qin T, Baud P, Zhou G, Xu T. Estimating in situ rock mass strength and elastic modulus of granite from the Soultz-sous-Forêts geothermal reservoir (France). Geotherm Energy. 2018;6:11. https://doi. org/10.1186/s40517-018-0096-1.

Violay M, Heap MJ, Acosta M, Madonna C. Porosity evolution at the brittle-ductile transition in the continental crust: implications for deep hydro-geothermal circulation. Sci Rep. 2017;7:7705. https://doi.org/10.1038/s41598-01708108-5.

Wang HF, Bonner BP, Carlson SR, Kowallis BJ, Heard HC. Thermal stress cracking in granite. J Geophys Res. 1989;94:1745. https://doi.org/10.1029/JB094iB02p01745.

Weaver RL. On diffuse waves in solid media. J Acoust Soc Am. 1982;71(6):1608-9. https://doi.org/10.1121/1.387816.

Wegler U, Lühr B-G. Scattering behaviour at Merapi volcano (Java) revealed from an active seismic experiment. Geophys 」 Int. 2001;145:579-92. https://doi.org/10.1046/j.1365-246x.2001.01390.x.

XUT, Zhou GL, Heap MJ, Zhu WC, Chen CF, Baud P. The influence of temperature on time-dependent deformation and failure in granite: a mesoscale modeling approach. Rock Mech Rock Eng. 2017;50:2345-64. https://doi.org/10.1007/ s00603-017-1228-9.

Yong C, Wang C. Thermally induced acoustic emission in Westerly Granite. Geophys Res Lett. 1980;7:1089-92. https://doi. org/10.1029/GL007i012p01089. 